\title{
Correlates of Physical Activity among Adults with Sight Loss in High-Income-Countries: A Systematic Review
}

\author{
Rosie K. Lindsay ${ }^{1}$, Francesco Di Gennaro ${ }^{2}{ }^{\circledR}$, Peter M. Allen ${ }^{1}$, Mark A. Tully ${ }^{3}{ }^{(D}$, Claudia Marotta ${ }^{4}{ }^{\circledR}$,

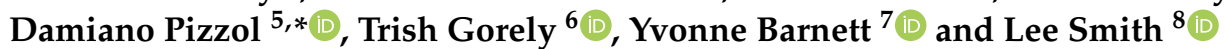

1 Vision and Hearing Sciences Research Centre, Anglia Ruskin University, Cambridge CB1 1PT, UK; rk1109@pgr.aru.ac.uk (R.K.L.); peter.allen@aru.ac.uk (P.M.A.)

2 Department of Biomedical Sciences and Human Oncology, Clinic of Infectious Diseases, University of Bari "Aldo Moro", 70124 Bari, Italy; cicciodigennaro@yahoo.it

3 Institute of Mental Health Sciences, School of Health Sciences, Ulster University, Newtownabbey BT37 0QB, UK; m.tully@ulster.ac.uk

4 General Directorate of Health Prevention, Ministry of Health, 00144 Rome, Italy; marotta.claudia@gmail.com

5 Clinical Board, Cigna Health Insurance, 00185 Rome, Italy

6 Centre for Health Science, Department of Nursing and Midwifery, University of the Highlands and Islands, Inverness IV2 3JH, UK; trish.gorely@uhi.ac.uk

7 School of Life Sciences, Faculty of Science and Engineering, Anglia Ruskin University, Cambridge CB1 1PT, UK; yvonne.barnett@aru.ac.uk

8 The Cambridge Centre for Sport and Exercise Science, Anglia Ruskin University, Cambridge CB1 1PT, UK; lee.smith@aru.ac.uk

\section{check for} updates

Citation: Lindsay, R.K.; Di Gennaro, F.; Allen, P.M.; Tully, M.A.; Marotta,

C.; Pizzol, D.; Gorely, T.; Barnett, Y.; Smith, L. Correlates of Physical Activity among Adults with Sight Loss in High-Income-Countries: A Systematic Review. Int. J. Environ. Res. Public Health 2021, 18, 11763. https: / / doi.org/10.3390/ ijerph182211763

Academic Editor: Paul B. Tchounwou

Received: 22 October 2021

Accepted: 7 November 2021

Published: 9 November 2021

Publisher's Note: MDPI stays neutral with regard to jurisdictional claims in published maps and institutional affiliations.

Copyright: (c) 2021 by the authors. Licensee MDPI, Basel, Switzerland. This article is an open access article distributed under the terms and conditions of the Creative Commons Attribution (CC BY) license (https:/ / creativecommons.org/licenses/by/ $4.0 /)$.
* Correspondence: damianopizzol8@gmail.com

\begin{abstract}
Background: Physical activity (PA) is essential for almost all facets of health; however, research suggests that PA levels among populations with sight loss are critically low. The aim of this review was to identify the correlates of PA among people with sight loss in high income countries, to inform future interventions and policies. Methods: MEDLINE, Web of Science, PsycINFO, SPORTDiscus, The British Journal of Visual Impairment, The Journal of Visual Impairment and grey literature were searched for studies which reported correlates of PA among adults with sight loss. The protocol is available from PROSPERO (CRD42020215596). Results: A total of 29 articles were eligible for review. Evidence from multiple studies reported that the vision impairment category, worse visual acuity, bilateral visual field loss, worse contrast sensitivity, those of the female gender, low self-efficacy, and environmental barriers were associated with lower levels of PA among populations with sight loss. Conclusions: Overall, correlates of PA among people with sight loss in high income countries are complex and vary across different population groups. Health professionals, eye care, and sight loss services should work together to identify people at risk of low PA, and provide a range of services and interventions to influence the modifiable factors that are associated with low PA.
\end{abstract}

Keywords: vision loss; visual impairment; physical activity; modifiable; non-modifiable; correlates

\section{Introduction}

Physical activity (PA) is defined as 'any movement produced by skeletal muscles that requires energy expenditure-including activities undertaken while working, playing, carrying out household chores, travelling, and engaging in recreational pursuits' [1]. The World Health Organisation (WHO) recommends that adults should engage in at least 150 min of moderate PA or 75 min of vigorous PA, or an equivalent combination of both intensities throughout the week, these recommendations are the same for adults with disabilities [2]. Moderate PA can be defined as PA performed at 3-6 times the intensity of rest, whilst vigorous PA can be defined as PA performed at $>6$ times the intensity of rest and moderate-vigorous PA (MVPA) is PA performed at $>3$ the intensity of rest [2]. Regular and sustained participation in MVPA is beneficial for almost every aspect of physical 
and mental health. Indeed, there is a large body of literature that shows that regular and sustained participation in MVPA can aid in the prevention of a plethora of chronic conditions [3].

At least 2.2 billion people worldwide have a near or distance visual impairment (VI) [4]. Visual impairment is classified into distance and near presenting vision impairment. A distance vision impairment ranges from mild (visual acuity worse than 6/12 to 6/18) to blindness (visual acuity worse than 3/60). A near vision impairment is defined as near visual acuity worse than N6 or M.08 at $40 \mathrm{~cm}$ [5]. In the UK, it is estimated that there are over 2 million people living with sight loss; this includes people with sight loss severe enough to be certified as visually impaired (sight impaired) or blind (severely sight impaired), as well as those with less severe sight loss. By 2050 the number of people living with sight loss in the UK is predicted to increase to over 4 million [6]. However, studies conducted in high income countries (HIC) have consistently reported that low levels of PA are associated with poorer vision $[7,8]$. For example, in a sample of older English adults, those who rated their eye-sight 'fair-poor' whilst wearing glasses or contact lenses were more than twice as likely to report being physically inactive than adults who reported having 'excellent vision' [9]. Low levels of PA are a concern as research has found that low levels of PA are associated with an increased risk of mortality in populations with sight loss [10-12].

Despite a global and growing population of people with sight loss, as well as the risks of low levels of PA, few studies have examined interventions aiming to promote PA among populations with sight loss. A systematic review of PA interventions for adults with VI found evidence that interventions can be beneficial, particularly for measures of balance and mobility [13]. However, in a subsequent meta-analysis that combined the results of four studies, the effect of PA interventions on mobility outcome measures were non-significant [13]. To inform the development of future interventions, it is important to first identify correlates of PA in those with sight loss.

Correlates can be either modifiable or non-modifiable. Modifiable correlates provide mechanisms to target for change (e.g., self-efficacy, accessible facilities), while nonmodifiable correlates determine the target population for a PA intervention (e.g., visually impaired versus blind, additional impairments versus no additional impairment, males versus females or young versus old). People with sight loss represent a diverse population, and people's experience of sight loss may depend on the severity of their sight loss, or the part of their sight which is impaired. For example, age-related macular degeneration predominantly affects central vision [14], whilst retinitis pigmentosa affects peripheral vision [15]; someone with either condition may have sight loss severe enough to be registered as VI, however both conditions affect sight differently. In addition, sight loss can occur across the sociodemographic spectrum, and sociodemographic factors and sight loss could intersect to influence PA either positively or negatively.

However, to our knowledge no attempt has been made to understand the complexities of the factors which could be associated with PA among people with sight loss, which is needed to determine targeted interventions. Therefore, the aim of this review was to identify modifiable and non-modifiable correlates of PA among people with sight loss in high income countries (HICs).

\section{Materials and Methods}

\subsection{Search Strategy}

The review followed the reporting guidelines outlined by the PRISMA checklist [16]. The protocol for the present review has been registered in the PROSPERO database (CRD42020215596). To identify studies which reported correlates with PA among populations with sight loss, electronic databases, reference lists, and grey literature were searched. We searched MEDLINE, Web of Science, PsycINFO, and SPORTDiscus for English language articles published from database inception to 1 October 2020. A subject librarian was consulted, and the search terms included terms related to physical activity AND correlates AND low vision (see Appendix A). The title and abstracts of all the search results 
were independently screened by two trained reviewers (RL and LS) for articles eligible for full text screening. The reference list of studies included in full text screening were also examined for eligible studies. The grey literature was searched from inception to November 2020 using the databases EThOS and Open Grey. The websites for the Royal National Institute of Blind People (RNIB), British Blind Sport, The Macular Society, Blind Veterans UK, Sight Savers, Guide Dogs for the Blind Association, Fight for Sight, SeeAbility, International Glaucoma Association (IGA), and World Sight Foundation were also searched for eligible studies. The British Journal of Visual Impairment, and The Journal of Visual Impairment and Blindness were also searched for the terms 'exercise', 'physical activity', and 'sport' in articles published from 2020-3 November 2021.

\subsection{Study Selection}

Studies were included if they were cross-sectional or longitudinal observational studies that met the following inclusion criteria: (1) included adults aged 18 and over, or the study performed a sub-group analysis of adults (age 18 and over). (2) Studies were conducted among participants with sight loss whilst wearing corrective devices, either measured by clinical assessment or self-reported sight loss. (3) Studies conducted in HICs (defined as countries identified by the World Bank as high income in 2020). (4) The dependent variable was a measure of PA engagement (e.g., how often and for how long a person goes to a sports club or walks for transport). (5) The significance level of the association between PA and an independent variable was examined, and a $p$-value was reported. The review excluded studies conducted among populations under the age of 18 , as correlates of PA among people under the age of 18 with sight loss is the focus of another review [17]. In addition, the review focused on correlates among HICs because these countries were more likely to be similar in terms of economic and social characteristics, therefore the results could be synthesized to inform interventions in HICs.

The exclusion criteria were: (1) Studies not written in the English language. (2) Studies which examined the relationship between PA and risk factors for sight loss or eye disease e.g., the relationship between ocular perfusion pressure and PA. (3) Studies which compared PA between populations with sight loss and populations without sight loss and did not report the factors associated with PA within the population with sight loss. There were no further restrictions applied to the population of study i.e., studies were included regardless of the gender, ethnicity and reported comorbidities of the population group.

The data were independently extracted by one reviewer (R.K.L), and then discussed with a second reviewer (L.S). The data extracted included: age (years), the gender of participants, the sample size, the PA measurement tool, the vision measurement tool, the eye disease examined (if applicable), the statistical test used, the country in which the participants were recruited from, the confounders controlled for when examining correlates of PA, and the main findings.

\section{Results}

The database search yielded 2854 results; of these citations, 792 duplicates were removed. The grey literature was also searched, and no eligible studies were found. The primary reason no eligible studies were found in the grey literature search was that no studies reported the significance level of the variables which were associated with PA participation. A search of key terms related to physical activity in the British Journal of Visual Impairment, and The Journal of Visual Impairment yielded 112 and 71 articles, respectively.

Following the abstract and title screening, and the subsequent full text screening, 29 studies were retained for the final review [7,18-45] (Figure 1). The data from eligible studies was extracted and reported in Table 1. 


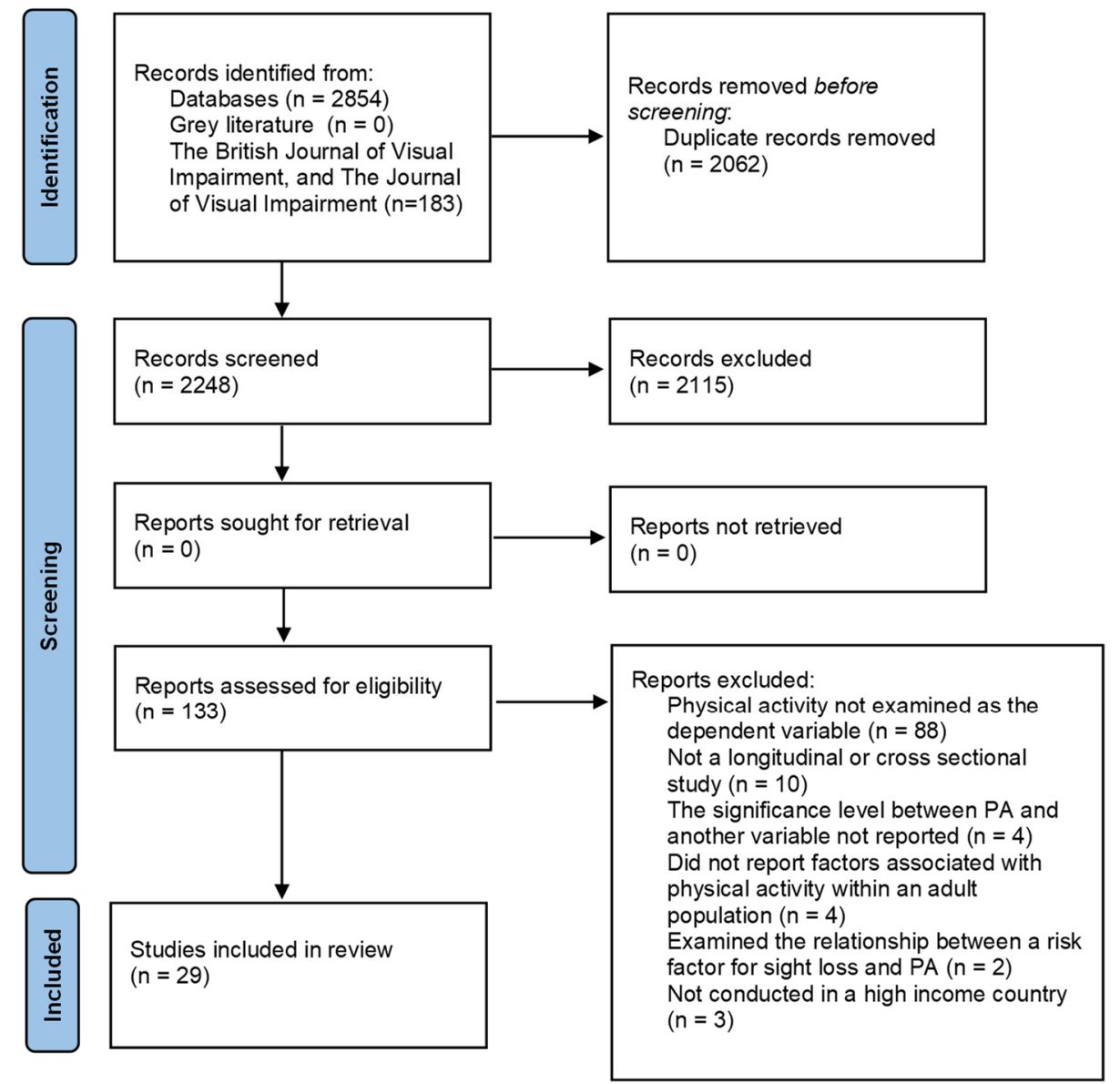

Figure 1. PRISMA flow diagram.

The Critical Appraisal Skills Programme (CASP) checklist [46] was completed for each study and informed the final quality assessment; the results are presented in Table 2. Out of all eligible studies, 4/29 were considered to be of a high quality, 14/29 studies were of a medium quality, and 11/29 of the studies were considered to be of a low quality. Studies were more likely to be considered of a higher quality if they had minimised the risk of selection bias, had recruited a sample size which allowed for reliable conclusions to be made regarding the statistical significance of associations, and controlled for confounding factors, such as age and gender, in the analysis. Studies which relied on objective measures of PA and included a clinical assessment of vision parameters to categorise participants were also more likely to be considered of a higher quality than studies which used self-reported methods of PA and sight loss. 
Table 1. Characteristics of eligible studies.

\begin{tabular}{|c|c|c|c|c|c|c|c|c|c|}
\hline Reference & $\begin{array}{c}\text { Population } \\
\text { Characteristics: Age } \\
\text { (Years), Gender. }\end{array}$ & Population: $(n)$ & $\begin{array}{l}\text { Physical Activity } \\
\text { Measurement Tool }\end{array}$ & $\begin{array}{l}\text { Vision Measurement } \\
\text { Tool }\end{array}$ & $\begin{array}{l}\text { Specific Eye Disease } \\
\text { Examined? }\end{array}$ & Statistical Test & Country & $\begin{array}{l}\text { Confounders } \\
\text { Controlled for }\end{array}$ & Main Findings \\
\hline $\begin{array}{l}\text { Barnett, A. et al. } \\
\text { (2016) [18] }\end{array}$ & $\begin{array}{c}\text { Mean age years (SD) } \\
\text { age: } 76(6) \\
\text { Male: } 34 \% \\
\text { Female: } 66 \%\end{array}$ & $\begin{array}{l}n=527 \\
\text { (participant s } \\
\text { with VI) }\end{array}$ & $\begin{array}{l}\text { IPAQ-SF (Chinese } \\
\text { version) }\end{array}$ & $\begin{array}{l}\text { VI was determined } \\
\text { using information from } \\
\text { clinical } \\
\text { health-problems } \\
\text { checklists obtained } \\
\text { from the Elderly } \\
\text { Health Center (EHC). } \\
\text { For participants } \\
\text { recruited outside of the } \\
\text { EHCs VI was } \\
\text { self-reported. }\end{array}$ & Glaucoma Cataracts & $\begin{array}{l}\text { The study examined if } \\
\text { VI had a moderating } \\
\text { effect on associations } \\
\text { between perceived } \\
\text { neighbourhood } \\
\text { characteristics and } \\
\text { physical activity } \\
\text { outcomes. }\end{array}$ & Hong Kong & $\begin{array}{l}\text { Socio demographics, } \\
\text { type of recruitment } \\
\text { centre, specific } \\
\text { diagnosed chronic } \\
\text { condition type, } \\
\text { number of other } \\
\text { medical conditions, } \\
\text { and other significant } \\
\text { perceived } \\
\text { neighbourhood } \\
\text { characteristics and } \\
\text { environment by } \\
\text { chronic condition } \\
\text { interaction effects. }\end{array}$ & $\begin{array}{c}\text { Land use mix-access to } \\
\text { services was positively } \\
\text { associated with } \\
\text { non-walking PA. Physica } \\
\text { barriers to walking were } \\
\text { negatively associated } \\
\text { with non-walking PA. } \\
\text { Weekly frequency and } \\
\text { minutes of } \\
\text { within-neighbourhood } \\
\text { walking for transport } \\
\text { was not significantly } \\
\text { associated with aesthetics } \\
\text { in participants with VI. }\end{array}$ \\
\hline $\begin{array}{l}\text { Black, A. et al. } \\
\text { (2011) [19] }\end{array}$ & $\begin{array}{c}\text { Mean age (SD) years: } \\
74.2(5.9) \\
\text { Female: } 47 \% \\
\text { Male: } 53 \%\end{array}$ & $n=74$ & $\begin{array}{c}\text { Self-reported. } \\
\text { Physical Activity } \\
\text { Scale for the Elderly } \\
\text { (PASE) }\end{array}$ & $\begin{array}{c}\text { VA: Standard } \\
\text { Bailey-Lovie } \\
\text { high-contrast } \\
\text { letter chart. } \\
\text { VF: Humphrey Field } \\
\text { Analyzer; model } \\
\text { HFA-II 750; Carl Zeiss } \\
\text { Meditec Inc., Dublin, } \\
\text { CA, USA). Monocular } \\
\text { 24-2 Swedish } \\
\text { Interactive Threshold } \\
\text { Algorithm } \\
\text { (SITA)-Standard } \\
\text { threshold tests. }\end{array}$ & Glaucoma patients. & $\begin{array}{l}\text { Multivariate regression } \\
\text { used to determine } \\
\text { which vision } \\
\text { parameters predicted } \\
\text { variations in PASE } \\
\text { scores. } \\
\text { Principal components } \\
\text { factors analysis with } \\
\text { varimax } \\
\text { transformation was } \\
\text { used to create three } \\
\text { vision factors which } \\
\text { were assessed in } \\
\text { multivariate analysis. }\end{array}$ & Australia & Age and gender & $\begin{array}{c}\text { PASE scores were } \\
\text { significantly associated } \\
\text { with contrast sensitivity } \\
\text { ( } \mathrm{r}=0.24) \text { and all of the } \mathrm{VH} \\
\text { measures. The } \\
\text { multivariate regression } \\
\text { which examined vision } \\
\text { factors and PA reported } \\
\text { 10.2\% of the variance in } \\
\text { PASE scores was } \\
\text { explained by vision } \\
\text { factors, contrast factor } \\
\text { was a significant } \\
\text { predictor of PASE scores, } \\
\text { whilst superior and } \\
\text { inferior field factors } \\
\text { were not. }\end{array}$ \\
\hline $\begin{array}{l}\text { Haegele, J. et al. } \\
\text { (2016) [20] }\end{array}$ & $\begin{array}{c}\text { Mean age years: } \\
47.04 \\
\text { Female: } 52.8 \% \\
\text { Male: } 47.2 \%\end{array}$ & $n=176$ & IPAQ-SF & $\begin{array}{l}\text { Self-reported visual } \\
\text { impairment } \\
\text { classification }\end{array}$ & Any & $\begin{array}{l}\text { Multiple regression } \\
\text { analysis with total } \\
\text { MET minutes per week } \\
\text { as the dependable } \\
\text { variable and } \\
\text { sociodemographic } \\
\text { variables as the } \\
\text { predictors. }\end{array}$ & USA & $\begin{array}{l}\text { Gender, ethnicity, VI } \\
\text { type, onset, years of VI, } \\
\text { K-12 education } \\
\text { mobility aid and } \\
\text { college education } \\
\text { included in multiple } \\
\text { regression. }\end{array}$ & $\begin{array}{l}\text { Gender was the only } \\
\text { significant predictor of } \\
\text { MET minutes per week } \\
(\beta=0.25, p<0.05) \text {, with } \\
\text { men reporting more MET } \\
\text { minutes than women. }\end{array}$ \\
\hline
\end{tabular}


Table 1. Cont.

\begin{tabular}{|c|c|c|c|c|c|c|c|c|c|}
\hline Reference & $\begin{array}{c}\text { Population } \\
\text { Characteristics: Age } \\
\text { (Years), Gender. }\end{array}$ & Population: $(n)$ & $\begin{array}{l}\text { Physical Activity } \\
\text { Measurement Tool }\end{array}$ & Vision Measurement Tool & $\begin{array}{l}\text { Specific Eye } \\
\text { Disease } \\
\text { Examined? }\end{array}$ & Statistical Test & Country & $\begin{array}{l}\text { Confounders } \\
\text { Controlled for }\end{array}$ & Main Findings \\
\hline $\begin{array}{l}\text { Haegele, J. et al. } \\
\text { (2017) [21] }\end{array}$ & $\begin{array}{c}\text { Mean age (SD) years: } \\
46.88(13.91) \\
\text { Female: } 54.3 \% \\
\text { Male: } 45.7 \%\end{array}$ & $n=92$ & IPAQ-SF & $\begin{array}{l}\text { Self-reported VI category. B1 } \\
\text { (blind), B2 (travel vision), and } \\
\text { B3 (legal blindness) }=28 .\end{array}$ & No & $\begin{array}{l}\text { Hierarchical multiple } \\
\text { regression analysis } \\
\text { with forced entry }\end{array}$ & USA & $\begin{array}{l}\text { Hierarchical multiple } \\
\text { regression analysis } \\
\text { controlled for vision } \\
\text { status, sex and age. }\end{array}$ & $\begin{array}{c}\text { The hierarchical } \\
\text { regression found vision } \\
\text { category }(p<0.001) \text { and } \\
\text { social support } \\
\text { significantly predicted } \\
\text { total METs }(p=0.037) \text { but } \\
\text { self-regulation }(p=0.094), \\
\text { sex }(p=0.069), \text { and age } \\
\text { did not. }\end{array}$ \\
\hline $\begin{array}{l}\text { Haegele, J. et al. } \\
\text { (2017) [23] }\end{array}$ & $\begin{array}{c}\text { Mean age (SD): } 47.5 \\
\text { (12.4) } \\
\text { Female: } 62.5 \% \\
\text { Male: } 37.5 \%\end{array}$ & $n=80$ & $\begin{array}{l}\text { Self-reported PA by } \\
\text { the IPAQ-SF }\end{array}$ & $\begin{array}{l}\text { Self-reported visual } \\
\text { impairment. Participants had } \\
\text { the option to select B1 (i.e., } \\
\text { Blind), B2 (i.e., travel vision), } \\
\text { or B3 (i.e., legal blindness). }\end{array}$ & No & $\begin{array}{l}\text { Spearman rank } \\
\text { correlation (to test } \\
\text { ordinal variables), } \\
\text { Pearson correlation (to } \\
\text { test continuous } \\
\text { variables). }\end{array}$ & USA & $\begin{array}{l}\text { No confounders } \\
\text { controlled for when } \\
\text { conducting Spearman } \\
\text { rank correlation } \\
\text { testing. }\end{array}$ & $\begin{array}{l}\text { Men reported higher } \\
\text { levels of PA than women } \\
(p=0.002) \text {. Level of } \\
\text { independence and PA } \\
\text { had a significant } \\
\text { relationship }(p=0.003), \\
\text { and health related quality } \\
\text { of life and PA }(p=0.010) \text {. } \\
\text { Sedentary behaviour and } \\
\text { PA were negatively } \\
\text { correlated ( } p=0.004) \text {. }\end{array}$ \\
\hline $\begin{array}{l}\text { Haegele, J. et al. } \\
\quad(2018)[24]\end{array}$ & $\begin{array}{c}\text { Mean age (SD): } \\
44.3(15.3) \\
\text { Female: } 65.2 \% \\
\text { Male: } 34.8 \%\end{array}$ & $n=147$ & IPAQ-SF & $\begin{array}{l}\text { Self-Report visual impairment } \\
\text { level. (B1, B2, B3) }\end{array}$ & No. & $\begin{array}{l}\text { Linear multiple } \\
\text { regression with forced } \\
\text { entry for all } \\
\text { independent variable } \\
\text { to explore impact } \\
\text { between variables and } \\
\text { MET-min/week. }\end{array}$ & USA & $\begin{array}{c}\text { Age, gender, VI } \\
\text { classification and } \\
\text { household income and } \\
\text { self-efficacy included } \\
\text { in the multiple } \\
\text { regression analysis, } \\
\text { MET-min/week was } \\
\text { the dependent } \\
\text { variable. }\end{array}$ & $\begin{array}{l}\text { In multiple regression } \\
\text { analysis self-efficacy was } \\
\text { the only variable that } \\
\text { reached significance as a } \\
\text { positive predictor of } \\
\text { MET-min/week } \\
(p=0.001) .\end{array}$ \\
\hline $\begin{array}{l}\text { Haegele, J. et al. } \\
\text { (2019) [25] }\end{array}$ & $\begin{array}{c}\text { Mean age (SD) years: } \\
44.77(15.3) \\
\text { Male: } 27.7 \% \\
\text { Female: } 72.3 \%\end{array}$ & $n=159$ & IPAQ-SF & $\begin{array}{l}\text { Self-reported VI classification } \\
\text { based on the United States } \\
\text { Association of Blind } \\
\text { Athletes system. }\end{array}$ & No. & $\begin{array}{c}\text { Hypothesised } \\
\text { structural model was } \\
\text { tested. }\end{array}$ & USA & $\begin{array}{l}\text { The structural model } \\
\text { predicting quality of } \\
\text { life, examined the } \\
\text { direct and indirect } \\
\text { paths predicting } \\
\text { self-efficacy for } \\
\text { exercise, physical } \\
\text { health and } \\
\text { psychological health } \\
\text { and MVPA. }\end{array}$ & $\begin{array}{l}\text { Self-efficacy for exercise } \\
\text { positively predicted } \\
\text { participants' weekly } \\
\text { MVPA }(\beta=0.26) .\end{array}$ \\
\hline
\end{tabular}


Table 1. Cont.

\begin{tabular}{|c|c|c|c|c|c|c|c|c|c|}
\hline Reference & $\begin{array}{c}\text { Population } \\
\text { Characteristics: Age } \\
\text { (Years), Gender. }\end{array}$ & Population: $(n)$ & $\begin{array}{l}\text { Physical Activity } \\
\text { Measurement Tool }\end{array}$ & Vision Measurement Tool & $\begin{array}{l}\text { Specific Eye } \\
\text { Disease } \\
\text { Examined? }\end{array}$ & Statistical Test & Country & $\begin{array}{l}\text { Confounders } \\
\text { Controlled for }\end{array}$ & Main Findings \\
\hline $\begin{array}{l}\text { Haegele, J. et al. } \\
\text { (2021) } \\
{[26]}\end{array}$ & $\begin{array}{c}\text { Mean age (SD) years: } \\
44.8(15.5) \\
\text { Male: } 43.6 \% \\
\text { Female: } 65.4 \%\end{array}$ & $n=182$ & IPAQ-SF & $\begin{array}{l}\text { Self-reported VI classification } \\
\text { based on the United States } \\
\text { Association of Blind Athletes } \\
\text { system }\end{array}$ & No & $\begin{array}{l}\text { Pearson } \\
\text { product-moment } \\
\text { correlation analysis. } \\
\text { Hierarchical } \\
\text { regression analyses }\end{array}$ & USA & $\begin{array}{l}\text { No confounders } \\
\text { controlled for } \\
\text { in-person product } \\
\text { moment correlation } \\
\text { analysis. } \\
\text { VI level, sex. Age, race, } \\
\text { education level., body } \\
\text { weight status, met } \\
\text { MVPA guideline, met } \\
\text { sedentary time } \\
\text { guideline, and met } \\
\text { sleep guidance were } \\
\text { included in } \\
\text { hierarchical } \\
\text { regression analyses. }\end{array}$ & $\begin{array}{l}\text { MVPA had a negative } \\
\text { correlation with BMI ( } p< \\
0.05 \text { ). The association } \\
\text { between MVPA, and age, } \\
\text { sedentary time, sleep and } \\
\text { depression was not } \\
\text { significant in correlation } \\
\text { analysis. } \\
\text { No significant association } \\
\text { was found between } \\
\text { meeting PA guidelines } \\
\text { and depression in } \\
\text { regression analysis. }\end{array}$ \\
\hline $\begin{array}{l}\text { Holbrook, E. et al. } \\
\quad(2009) \text { [27] }\end{array}$ & $\begin{array}{l}\text { Age range: } 18-60 \\
\text { Male: } 60 \% \\
\text { Female: } 40 \%\end{array}$ & $n=25$ & $\begin{array}{l}\text { Step Activity } \\
\text { Monitor (SAM; } \\
\text { Cyma, Seattle, WA; } \\
\text { Model SW3). }\end{array}$ & $\begin{array}{l}\text { Self-report VI severity based } \\
\text { on the ICD classification }\end{array}$ & No & $\begin{array}{l}3 \text { (mild, moderate, } \\
\text { severe) } \times 2 \text { (male, } \\
\text { female) ANOVAs. }\end{array}$ & USA & $\begin{array}{l}\text { The data was stratified } \\
\text { by VI severity and by } \\
\text { gender. }\end{array}$ & $\begin{array}{l}\text { No interaction between } \\
\text { the severity of VI and } \\
\text { gender for four of the PA } \\
\text { variables: average daily } \\
\text { step counts, }(p=0.35) \\
\text { percentage of time at low } \\
\text { activity, }(p=0.36) \\
\text { percentage of time at } \\
\text { moderate activity } \\
(p=0.93 .)\end{array}$ \\
\hline $\begin{array}{l}\text { Holbrook, K. et al. } \\
\text { (2013) [28] }\end{array}$ & $\begin{array}{c}\text { Mean age (SD) years: } \\
45.9(11 .) . \\
\text { Female: } 61.3 \% \\
\text { Male: } 38.7 \%\end{array}$ & $n=31$ & $\begin{array}{l}\text { Pedometer (Orbxy, } \\
\text { Electronics Model } \\
\text { 6310610, Concord, } \\
\text { Canada) }\end{array}$ & $\begin{array}{l}\text { Self-reported VI classification } \\
\text { based on the International } \\
\text { Statistical Classification for } \\
\text { Disease schematic. }\end{array}$ & No & $\begin{array}{l}\text { One-way repeated } \\
\text { measures ANOVA } \\
\text { Generalizability } \\
\text { theory analysis }\end{array}$ & USA & $\begin{array}{c}\text { PA stability was } \\
\text { assessed across } \\
\text { varying VI severity. }\end{array}$ & $\begin{array}{c}\text { No difference in daily } \\
\text { step activity across the } \\
\text { days of the week in the } \\
\text { whole sample }(p=0.633) \text {. } \\
4 \text { days monitoring was } \\
\text { sufficient for a reliable } \\
\text { measure of PA among } \\
\text { people with } \\
\text { mild-moderate VI and } \\
9 \text { days for people with } \\
\text { severe VI. }\end{array}$ \\
\hline
\end{tabular}


Table 1. Cont.

\begin{tabular}{|c|c|c|c|c|c|c|c|c|c|}
\hline Reference & $\begin{array}{c}\text { Population } \\
\text { Characteristics: Age } \\
\text { (Years), Gender. }\end{array}$ & Population: $(n)$ & $\begin{array}{l}\text { Physical Activity } \\
\text { Measurement Tool }\end{array}$ & Vision Measurement Tool & $\begin{array}{l}\text { Specific Eye } \\
\text { Disease } \\
\text { Examined? }\end{array}$ & Statistical Test & Country & $\begin{array}{l}\text { Confounders } \\
\text { Controlled for }\end{array}$ & Main Findings \\
\hline $\begin{array}{l}\text { Inoue, S. et al. } \\
\text { (2018) [29] }\end{array}$ & $\begin{array}{c}\text { Mean age (SD, } \\
\text { range) years: } \\
69.6(14.5,20-93) \\
\text { Male: } 118(54.9 \%)\end{array}$ & $n=215$ & IPAQ-Japan & $\begin{array}{l}\text { BCVA was assessed using } \\
\text { clinical measures. }\end{array}$ & No & $\begin{array}{c}\text { Univariate and } \\
\text { multivariate ordinal } \\
\text { logistic regression } \\
\text { analysis to assess the } \\
\text { association between } \\
\text { physical activity and } \\
\text { variables. }\end{array}$ & Japan & $\begin{array}{l}\text { Sex, age, VFQ- } 25 \text { score, } \\
\text { BCVA in the better eye, } \\
\text { and BCVA in the worse } \\
\text { eye, systemic } \\
\text { comorbidity, and BMI } \\
\text { included in } \\
\text { multivariate models. }\end{array}$ & $\begin{array}{c}\text { Multivariate ordinal } \\
\text { logistic regression } \\
\text { analysis reported PA was } \\
\text { significantly associated } \\
\text { with VFQ-25 score } \\
(p<0.001) \text { and BCVA in } \\
\text { the worse eye }(p=0.01) \\
\text { but not BCVA in } \\
\text { better eye. } \\
\text { Sex, age, systemic } \\
\text { comorbidity, and BMI } \\
\text { was not associated with } \\
\text { PA in univariate or } \\
\text { multivariate models. }\end{array}$ \\
\hline $\begin{array}{l}\text { Jaarsma, E. et al. } \\
\text { (2014) [30] }\end{array}$ & $\begin{array}{c}\text { Mean age (SD) years: } \\
49.1(17.9) \\
\text { Female: } 52 \% \\
\text { Male: } 48 \%\end{array}$ & $n=648$ & Questionnaire. & $\begin{array}{l}\text { Self-reported VI category } \\
\text { based on ICD-10 }\end{array}$ & No & $\begin{array}{l}\text { Logistic regression } \\
\text { (method enter) which } \\
\text { included all variables } \\
\quad \text { related to sports } \\
\text { participation }(p \leq 0.1) .\end{array}$ & $\begin{array}{l}\text { The Nether- } \\
\text { lands }\end{array}$ & $\begin{array}{l}\text { Education, use of } \\
\text { white cane, use of } \\
\text { computer software, } \\
\text { having a guide dog, } \\
\text { disability (experienced } \\
\text { as a barrier), cost as a } \\
\text { barrier, lack of } \\
\text { peers/buddies as a } \\
\text { barrier, and gender } \\
\text { were entered as } \\
\text { predictors of sports } \\
\text { participation in the } \\
\text { logistic regression. }\end{array}$ & $\begin{array}{l}\text { The significant factors } \\
\text { predicting sports } \\
\text { participation were } \\
\text { education, disability } \\
\text { (experienced as a barrier), } \\
\text { costs, lack of } \\
\text { peer/buddies and use of } \\
\text { computer software. }\end{array}$ \\
\hline $\begin{array}{l}\text { Jones, G. et al. } \\
\text { (2010) [31] }\end{array}$ & 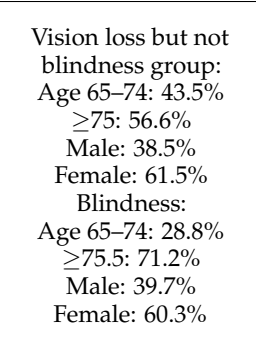 & $\begin{array}{l}\text { Normal sight: } n \\
\quad=33,497 \\
\text { Blind: } n=477 \\
\text { Vision loss but } \\
\text { not blindness: } n \\
=6721\end{array}$ & $\begin{array}{l}\text { Self-reported PA: } \\
\text { Respondents were } \\
\text { deemed physically } \\
\text { inactive if they } \\
\text { reported no regular } \\
\text { weekly exercise. }\end{array}$ & $\begin{array}{l}\text { Individuals who reported } \\
\text { trouble seeing, even with } \\
\text { glasses or contact lenses were } \\
\text { classified as having vision loss. } \\
\text { Participants were asked if } \\
\text { they were blind or could not } \\
\text { see at all. This was the case } \\
\text { definition of blindness. }\end{array}$ & No & Logistic regression & USA & $\begin{array}{l}\text { Age, sex, } \\
\text { race/ethnicity, income, } \\
\text { education, marital } \\
\text { status, and correlated } \\
\text { health behaviours. }\end{array}$ & $\begin{array}{c}\text { There was a strong } \\
\text { association between } \\
\text { physical inactivity and } \\
\text { severity of sight loss. } \\
75.9 \% \text { of adults with } \\
\text { blindness did not exercise } \\
\text { weekly (Adjusted odds } \\
\text { ratio }=2.24 \text { ), compared to } \\
61.2 \% \text { of adults with } \\
\text { vision loss and not } \\
\text { blindness (Adjusted odds } \\
\text { ratio = 1.25). }\end{array}$ \\
\hline $\begin{array}{l}\text { Łabudzki, T. et al. } \\
\text { (2013) [32] }\end{array}$ & $\begin{array}{c}\text { Mean age (SD): } 38 \\
( \pm 12.1) \\
\text { Female: } 52.4 \% \\
\text { Male: } 47.6 \%\end{array}$ & $n=82$ & IPAQ-SF & $\begin{array}{l}\text { Self-reported prior medical } \\
\text { diagnosis of VI (significant, } \\
\text { moderate or light } \\
\text { impairment). }\end{array}$ & No. & $\begin{array}{l}\text { Chi- square test for } \\
\text { gender difference. }\end{array}$ & Poland & $\begin{array}{l}\text { None controlled for } \\
\text { when testing for } \\
\text { differences in PA } \\
\text { between genders. }\end{array}$ & $\begin{array}{l}\text { No difference in PA in } \\
\text { relation to gender (Chi } \\
\text { square }=0.256, p=0.88) \text {. }\end{array}$ \\
\hline
\end{tabular}


Table 1. Cont.

\begin{tabular}{|c|c|c|c|c|c|c|c|c|c|}
\hline Reference & $\begin{array}{c}\text { Population } \\
\text { Characteristics: Age } \\
\text { (Years), Gender. }\end{array}$ & Population: $(n)$ & $\begin{array}{l}\text { Physical Activity } \\
\text { Measurement Tool }\end{array}$ & Vision Measurement Tool & $\begin{array}{c}\text { Specific Eye } \\
\text { Disease } \\
\text { Examined? }\end{array}$ & Statistical Test & Country & $\begin{array}{l}\text { Confounders } \\
\text { Controlled for }\end{array}$ & Main Findings \\
\hline $\begin{array}{l}\text { Lee M. et al. } \\
\text { (2014) [33] }\end{array}$ & $\begin{array}{l}\text { Age groups: } \mathrm{n} \\
\text { 20s: } 20 \\
30 \mathrm{~s}: 16 \\
40 \mathrm{~s}: 23 \\
50 \mathrm{~s}: 52 \\
>00 \mathrm{~s}: 33 \\
\text { Female: } 66.6 \% \\
\text { Male: } 33.3 \%\end{array}$ & $\begin{array}{l}n=144 \text { with } \\
\text { VI } / \text { blind. }\end{array}$ & PASIPD & Self-report VA & No & $\begin{array}{c}\text { Descriptive statistics } \\
\text { used to screen the data. } \\
\text { Pearson Product } \\
\text { Moment Correlations } \\
\text { (to examine } \\
\text { relationship between } \\
\text { PA participation and } \\
\text { PA barriers). }\end{array}$ & USA & $\begin{array}{l}\text { Confounders not } \\
\text { controlled for when } \\
\text { examining the } \\
\text { relationship between } \\
\text { PA and self-reported } \\
\text { barriers to PA. }\end{array}$ & $\begin{array}{l}\text { PA in the normal weight } \\
\text { group was higher than in } \\
\text { the overweight /obese } \\
\text { group }(\mathrm{t}=2.09, p<0.04) \text {. } \\
\text { A significant correlation } \\
(\mathrm{r}=0.41) \text { was reported } \\
\text { between PA levels and } \\
\text { the logit of reported } \\
\text { barriers to PA. }\end{array}$ \\
\hline $\begin{array}{l}\text { Marmeleira, J. } \\
\text { et al. (2014) [35] }\end{array}$ & $\begin{array}{c}\text { Mean age (SD) years: } \\
47.4(11.3) \\
\text { Male: } 41 \\
\text { Female: } 22\end{array}$ & $n=63$ & $\begin{array}{l}\text { Accelerometery(model } \\
\text { GT1M; ActiGraph, } \\
\text { Fort Walton } \\
\text { Beach, FL) }\end{array}$ & $\begin{array}{l}\text { People who were declared as } \\
\text { legally blind by the } \\
\text { Associatjao dos } \\
\text { Cegos e Amblfopes de } \\
\text { Portugal (The main } \\
\text { Portuguese association for } \\
\text { people with a VI). }\end{array}$ & Any & $\begin{array}{c}\text { Independent sample t } \\
\text { test or the } \\
\text { nonparametric } \\
\text { Mann-Whitney U test } \\
\text { were used to compare } \\
\text { PA between genders } \\
\text { and } \\
\text { congenital/acquired } \\
\text { blindness groups. } \\
\text { Chi-square test } \\
\text { compared the } \\
\text { proportion of } \\
\text { participants who } \\
\text { achieved the } \\
\text { recommended levels of } \\
\text { PA by gender. } \\
\text { One-way ANOVA } \\
\text { compared PA between } \\
\text { BMI categories. } \\
\text { Pearson's correlation } \\
\text { test relationship } \\
\text { between physical } \\
\text { activity and age. }\end{array}$ & Portugal & Not controlled for. & $\begin{array}{l}\text { No significant gender } \\
\text { difference in any of the } \\
\text { PA variables. No } \\
\text { differences across PA } \\
\text { variables across BMI } \\
\text { categories, age, age of } \\
\text { blindness onset, or when } \\
\text { comparing PA between } \\
\text { people with congenital or } \\
\text { acquired blindness. }\end{array}$ \\
\hline
\end{tabular}


Table 1. Cont

\begin{tabular}{|c|c|c|c|c|c|c|c|c|c|}
\hline Reference & $\begin{array}{c}\text { Population } \\
\text { Characteristics: Age } \\
\text { (Years), Gender. }\end{array}$ & Population: $(n)$ & $\begin{array}{l}\text { Physical Activity } \\
\text { Measurement Tool }\end{array}$ & Vision Measurement Tool & $\begin{array}{l}\text { Specific Eye } \\
\text { Disease } \\
\text { Examined? }\end{array}$ & Statistical Test & Country & $\begin{array}{l}\text { Confounders } \\
\text { Controlled for }\end{array}$ & Main Findings \\
\hline $\begin{array}{l}\text { McMullan, I. et al. } \\
\text { (2020) [7] }\end{array}$ & $\begin{array}{c}\text { Mean age years: } \\
63.57 \\
\text { Female: } 55 \% \\
\text { Male: } 45 \%\end{array}$ & $n=8255$ & IPAQ-SF & $\begin{array}{l}\text { Self-Report. Participants were } \\
\text { asked 'Is your eyesight (using } \\
\text { glasses or corrective contact } \\
\text { lenses) excellent, very good, } \\
\text { good, fair, or poor?' }\end{array}$ & No & Path analysis & $\begin{array}{l}\text { Republic of } \\
\text { Ireland }\end{array}$ & $\begin{array}{l}\text { Age, marital status, } \\
\text { sex, self-reported } \\
\text { health, education, } \\
\text { employment, } \\
\text { depression, history of } \\
\text { high blood pressure, } \\
\text { eye disease, diabetes, } \\
\text { and cardiovascular } \\
\text { disease, and disability } \\
\text { of activities of } \\
\text { daily living. }\end{array}$ & $\begin{array}{l}\text { Self-reported sight loss } \\
\text { did not directly affect PA. } \\
\text { However, PA had a } \\
\text { cumulative effect on } \\
\text { future PA, via its effect on } \\
\text { vision over } 6 \text { years. }\end{array}$ \\
\hline $\begin{array}{l}\text { Montarzino, A. } \\
\text { et al. (2007) [36] }\end{array}$ & $\begin{array}{c}\text { Mean age (SD): } \\
80.15(8.2) \\
\text { Female: } 67 \% \\
\text { Male: } 33 \%\end{array}$ & $n=66$ & Travel questionnaire. & Recruited from an eye clinic. & Any & $\begin{array}{l}\text { Kruskal Wallis 1-way } \\
\text { analysis of variance } \\
\text { was carried out to } \\
\text { identify significant } \\
\text { differences in walking } \\
\text { distance across all } \\
\text { acuity ranges at } \\
p<0.01 . \\
\text { Kruskal Wallis } \\
\text { ANOVA was run to } \\
\text { test variables were } \\
\text { significant } \\
\text { discriminators of } \\
\text { walking distance. The } \\
\text { significant variables } \\
\text { were then placed in a } \\
\text { binary logistic } \\
\text { regression to predict } \\
\text { walking distance. }\end{array}$ & $\begin{array}{c}\text { UK } \\
\text { (Scotland) }\end{array}$ & $\begin{array}{c}\text { Regression tree } \\
\text { analysis included age, } \\
\text { visual acuity and safety } \\
\text { concerns as predictors } \\
\text { of walking distance in } \\
\text { the model and } \\
\text { prioritised their } \\
\text { importance. }\end{array}$ & $\begin{array}{l}\text { The main restrictions on } \\
\text { walking are the age of the } \\
\text { participant, vision in the } \\
\text { better and worse eyes, } \\
\text { and feelings of safety, } \\
\text { however these factors } \\
\text { varied by age. }\end{array}$ \\
\hline $\begin{array}{l}\text { Nguyen, A et al. } \\
\text { (2015) [37] }\end{array}$ & $\begin{array}{c}\text { Controls: } \\
\text { Age, median (IQR) } \\
\text { 69.4 (65.2, } 72.8) \\
\text { Female: } 61.7 \% \\
\text { Glaucoma: } \\
\text { Age, median (IQR) } \\
70.4(66.4,74.5) \\
\text { Female: } 53 \% \\
\text { AMD: } \\
\text { Age, median (IQR) } \\
\text { 75.8 years }(71.0,78.3) \\
\text { Female: } 57.1 \%\end{array}$ & $\begin{array}{c}\text { Controls: } \\
n=59 \\
\text { Glaucoma: } \\
n=83 \\
\text { AMD: } n=58\end{array}$ & $\begin{array}{c}\text { Accelerometer } \\
\text { (Actical; Respironics, } \\
\text { Inc., Adover, } \\
\text { MA, USA) }\end{array}$ & $\begin{array}{l}\text { VF: Humphrey 24-2 VF } \\
\text { testing (Carl Zeiss Meditec, } \\
\text { Dublin, CA). } \\
\text { VA: ETDRS chart }\end{array}$ & $\begin{array}{l}\text { Glaucoma and } \\
\text { AMD }\end{array}$ & $\begin{array}{l}\text { Separate univariate } \\
\text { negative binomial } \\
\text { analyses were } \\
\text { performed with MVPA } \\
\text { as the dependent } \\
\text { variable to identify } \\
\text { covariates to be further } \\
\text { explored in } \\
\text { multivariate analyses. }\end{array}$ & USA & $\begin{array}{l}\text { Covariates included in } \\
\text { model } 1 \text { multivariate } \\
\text { analysis were CS, Age, } \\
\text { Sex, Race, Education, } \\
\text { and Comorbidity as } \\
\text { independent variables. } \\
\text { In model } 2 \text { fear of } \\
\text { falling was included as } \\
\text { a possible mediator } \\
\text { between CS } \\
\text { and MVPA. }\end{array}$ & $\begin{array}{l}\text { For participants with } \\
\text { AMD, the association } \\
\text { between CS and PA was } \\
\text { no longer significant once } \\
\text { fear of falling was added } \\
\text { to the model ( } p=0.53) \text {. } \\
\text { For participants with } \\
\text { glaucoma related VI, VF } \\
\text { loss remained a } \\
\text { statistically significant } \\
\text { predictor of physical } \\
\text { activity once fear of } \\
\text { falling was added to the } \\
\text { model }(p<0.01) .\end{array}$ \\
\hline
\end{tabular}


Table 1. Cont.

\begin{tabular}{|c|c|c|c|c|c|c|c|c|c|}
\hline Reference & $\begin{array}{c}\text { Population } \\
\text { Characteristics: Age } \\
\text { (Years), Gender. }\end{array}$ & Population: $(n)$ & $\begin{array}{l}\text { Physical Activity } \\
\text { Measurement Tool }\end{array}$ & Vision Measurement Tool & $\begin{array}{c}\text { Specific Eye } \\
\text { Disease } \\
\text { Examined? }\end{array}$ & Statistical Test & Country & $\begin{array}{l}\text { Confounders } \\
\text { Controlled for }\end{array}$ & Main Findings \\
\hline $\begin{array}{l}\text { Ramulu, P. et al. } \\
\text { (2012) [38] }\end{array}$ & $\begin{array}{l}\text { No glaucoma: } \\
\text { Age: } 69.3 \\
\text { Female (\%): } 62.1 \\
\text { Glaucoma: } \\
\text { Age: } 70.3 \\
\text { Female: } 54.2 \%\end{array}$ & $\begin{array}{c}\text { No glaucoma: } \\
n=58 \\
\text { Glaucoma: } \\
n=83\end{array}$ & $\begin{array}{c}\text { Omnidirectional } \\
\text { accelerometer } \\
\text { (Actical; Respironics, } \\
\text { Inc., Andover, } \\
\text { MA, USA). }\end{array}$ & $\begin{array}{l}\text { VA: ETDRS chart. } \\
\text { CS: Pelli-Robson chart (under } \\
\text { binocular conditions) } \\
\text { VF: Humphrey 24-2 VF } \\
\text { testing (Carl Zeiss Meditec, } \\
\text { Dublin, CA). }\end{array}$ & Glaucoma & $\begin{array}{c}\text { Univariate analysis } \\
\text { and Multivariate } \\
\text { analysis negative } \\
\text { binomial regression } \\
\text { models. }\end{array}$ & USA & $\begin{array}{l}\text { Variables included in } \\
\text { multivariate analysis: } \\
\text { Glaucoma (present), } \\
\text { severe Glaucoma } \\
\text { (present), visual field, } \\
\text { age, race, gender, } \\
\text { education, } \\
\text { comorbidities, } \\
\text { depressive symptoms, } \\
\text { BMI, cognitive ability. }\end{array}$ & $\begin{array}{l}\text { When the extent of VF } \\
\text { loss, visual acuity, and } \\
\text { contrast sensitivity were } \\
\text { included in the same } \\
\text { multivariate models, only } \\
\text { VF loss remained } \\
\text { predictive of either } \\
\text { MVPA or steps ( }(p<0.01 \\
\text { for both). }\end{array}$ \\
\hline $\begin{array}{l}\text { Ramulu, P. et al. } \\
\text { (2019) [39] }\end{array}$ & $\begin{array}{c}\text { Age mean (SD): } \\
70.7(7.6) \\
\text { Female: } 48 \% \\
\text { Male: } 52 \%\end{array}$ & $n=230$ & $\begin{array}{c}\text { Accelerometer } \\
\text { (Actical, Respironics } \\
\text { Inc., Murrysville, PA, } \\
\text { USA) }\end{array}$ & $\begin{array}{l}\text { VF: Humphrey Field Analyzer } \\
\text { 24-2 test (Carl Zeiss Meditec, } \\
\text { Inc., Dublin, CA, USA) } \\
\text { VA: ETDRS chart } \\
\text { CS: MARS chart }\end{array}$ & Glaucoma & $\begin{array}{l}\text { Univariate and } \\
\text { multivariable negative } \\
\text { binomial models. }\end{array}$ & USA & $\begin{array}{l}\text { Age, race, gender, } \\
\text { number of comorbid } \\
\text { illnesses, and } \\
\text { polypharmacy. }\end{array}$ & $\begin{array}{c}\text { In multivariable models, } \\
\text { worse integrated visual } \\
\text { field sensitivity, older age, } \\
\text { female gender, } \\
\text { African-American race } \\
\text { and polypharmacy was } \\
\text { associated with less daily } \\
\text { steps }(p \leq 0.05 \text { for all) } \\
\text { greater comorbid illness } \\
\text { was not associated with } \\
\text { less daily steps }(p=0.41) \text {. }\end{array}$ \\
\hline $\begin{array}{l}\text { Sengupta, N. et al. } \\
\text { (2015) [40] }\end{array}$ & $\begin{array}{c}\text { Control group: } \\
\text { Age mean: } 69.3(5.3) \\
\text { Female: } 62.7 \% \\
\text { AMD group: } \\
\text { Age mean: } 74.4(5.2) \\
\text { Female: } 57.9 \%\end{array}$ & $\begin{array}{c}\text { AMD patients: } \\
n=57 \\
\text { Controls with } \\
\text { normal vision: } \\
n=59\end{array}$ & $\begin{array}{c}\text { Accelerometer } \\
\text { (Actical; Respironics, } \\
\text { Inc., Andover, MA, } \\
\text { USA) }\end{array}$ & $\begin{array}{l}\text { VA: ETDRS chart } \\
\text { CS: Pelli-Robson chart }\end{array}$ & AMD & $\begin{array}{c}\text { Univariate and } \\
\text { multivariate negative } \\
\text { binomial regression } \\
\text { models. }\end{array}$ & USA & $\begin{array}{c}\text { Multivariate negative } \\
\text { binomial regression } \\
\text { models adjusted for } \\
\text { age, gender, race, } \\
\text { comorbidities, and } \\
\text { education. }\end{array}$ & $\begin{array}{c}\text { A significant } \\
\text { dose-dependent } \\
\text { relationship was } \\
\text { observed between worse } \\
\text { clinical measures of } \\
\text { vision and daily MVPA } \\
\text { and daily steps }(p<0.05) \text {. }\end{array}$ \\
\hline $\begin{array}{l}\text { Shakarchi, A. et al. } \\
\text { (2019) [41] }\end{array}$ & $\begin{array}{c}\text { Age mean }( \pm S D): \\
70( \pm 6.8) \\
\text { Female: } 53 \% \\
\text { Male: } 47 \%\end{array}$ & $n=151$ & $\begin{array}{c}\text { Accelerometer, } \\
\text { (Actical, Respironics, } \\
\text { Inc., Murrysville, PA, } \\
\text { USA) }\end{array}$ & $\begin{array}{c}\text { VA: ETDRS chart } \\
\text { CS: MARS chart } \\
\text { VF: Humphrey Field Analyzer } \\
\text { 24-2 test (Carl Zeiss Meditec, } \\
\text { Inc., Dublin, CA, USA) } \\
\text { AULCSF: Estimated from the } \\
\text { quick CS function (qCSF) test } \\
\text { (Adaptive Sensory } \\
\text { Technology, San Diego, } \\
\text { CA, USA) } \\
\text { Colour vision: } \\
\text { Hardy-Rand-Rittler (OttLite } \\
\text { Technology, Tampa, FL, USA) } \\
\text { Stereoacuity: Distance Randot } \\
\text { Stereotest (Stereo Optical, } \\
\text { Chicago, IL, USA). } \\
\text { ViN: Pelli-Levi Dual } \\
\text { Acuity Chart }\end{array}$ & Glaucoma & $\begin{array}{c}\text { Likelihood ratio testing } \\
\text { determined tested } \\
\text { association between PA } \\
\text { and vision parameters. } \\
\text { Dominance analysis } \\
\text { determined the relative } \\
\text { importance of the } \\
\text { various visual } \\
\text { parameters. }\end{array}$ & USA & $\begin{array}{l}\text { Age, sex, race, marital } \\
\text { status, living } \\
\text { arrangements, } \\
\text { employment status, } \\
\text { and education. } \\
\text { Polypharmacy } \\
\text { (defined as having five } \\
\text { or more non-eyedrop } \\
\text { prescription) and } \\
\text { comorbidities index. }\end{array}$ & $\begin{array}{l}\text { Vision parameters } \\
\text { significantly predicted } \\
\text { daily steps } p=0.01 \text {. The } \\
\text { dominant predictor of } \\
\text { differences in daily steps } \\
\text { was AULCF. }\end{array}$ \\
\hline
\end{tabular}


Table 1. Cont.

\begin{tabular}{|c|c|c|c|c|c|c|c|c|c|}
\hline Reference & $\begin{array}{c}\text { Population } \\
\text { Characteristics: Age } \\
\text { (Years), Gender. }\end{array}$ & Population: $(n)$ & $\begin{array}{l}\text { Physical Activity } \\
\text { Measurement Tool }\end{array}$ & Vision Measurement Tool & $\begin{array}{l}\text { Specific Eye } \\
\text { Disease } \\
\text { Examined? }\end{array}$ & Statistical Test & Country & $\begin{array}{l}\text { Confounders } \\
\text { Controlled for }\end{array}$ & Main Findings \\
\hline $\begin{array}{l}\text { Starkoff, B. et al. } \\
\text { (2017) [42] }\end{array}$ & $\begin{array}{c}\text { Mean age (SD) years: } \\
36.1(13.9) \\
\text { Men: } 48.7 \% \\
\text { Women: } 51.3 \%\end{array}$ & $n=115$ & IPAQ-SF & $\begin{array}{l}\text { VI was self-report } \\
\text { classification based on the } \\
\text { International Blind Sports } \\
\text { Federation and US association } \\
\text { of blind athletes guidelines } \\
\text { (B1, B2, B3, B4). }\end{array}$ & No. & $\begin{array}{l}\text { One-way ANOVAs to } \\
\text { assess PA differences } \\
\text { between gender, BMI } \\
\text { and extent of VI. } \\
\text { To differentiate } \\
\text { between blind and VI } \\
\text { participants a } \\
\text { 2X4 ANOVA was used } \\
\text { to assess differences in } \\
\text { PA between gender } \\
\text { and BMI. }\end{array}$ & USA & $\begin{array}{c}\text { Data was stratified by } \\
\text { different types of PA } \\
\text { (walking, moderate PA, } \\
\text { MVPA and vigorous } \\
\text { PA) }\end{array}$ & $\begin{array}{l}\text { Males engaged in more } \\
\text { moderate PA than } \\
\text { females }(p=0.008) . \\
\text { Walk time was } \\
\text { significantly greater in } \\
\text { participants who were } \\
\text { visually impaired } \\
\text { compared to those who } \\
\text { were blind ( } p=0.021) \text {. } \\
\text { Overweight participants } \\
\text { engaged in more } \\
\text { vigorous PA compared to } \\
\text { normal-weight } \\
\text { participants }(p=0.020) .\end{array}$ \\
\hline $\begin{array}{l}\text { Subhi, Y. et al. } \\
\text { (2016) [43] }\end{array}$ & $\begin{array}{c}\text { No AMD: } \\
\text { Mean age (SD): } \\
70.5 \text { (7.5) } \\
\text { Female: } 63 \% \\
\text { Male: } 37 \% \\
\text { Early AMD } \\
\text { Mean age (SD): } \\
77.1(5.6) \\
\text { Female: } 52 \% \\
\text { Male: } 48 \% \\
\text { Late AMD: } \\
\text { Mean age (SD): } \\
75.9(7.6) \\
\text { Female: } 64 \% \\
\text { Male: } 36 \%\end{array}$ & $\begin{array}{c}\text { No AMD: } n=68 \\
\text { Early AMD: } n= \\
25 \\
\text { Late AMD: } n= \\
103\end{array}$ & $\begin{array}{c}\text { Self-report } \\
\text { questionnaire. }\end{array}$ & $\begin{array}{l}\text { BCVA in each eye was } \\
\text { measure using Early } \\
\text { Treatment of the Diabetic } \\
\text { Retinopathy study chart. }\end{array}$ & AMD & $\begin{array}{l}\text { Chi-Square test or } \\
\text { Fisher's exact test } \\
\text { when numbers } \\
\text { were small. }\end{array}$ & Denmark & $\begin{array}{l}\text { Participants were age } \\
\text { matched with healthy } \\
\text { participants with no } \\
\text { AMD. }\end{array}$ & $\begin{array}{c}\text { No difference in between } \\
\text { participants at different } \\
\text { stages of AMD. } \\
\text { People with worse BVCA } \\
\text { in best-seeing eye and } \\
\text { worse-seeing eye were } \\
\text { more likely to report: not } \\
\text { engaging in PA weekly, } \\
\text { to report engaging in } \\
\text { vigorous PA }<1 \text { times a } \\
\text { week compared to } \geq 1 \text { a } \\
\text { week and walking } \leq \\
10 \text { stairs steps daily } \\
\text { compared to } 11-50 \text { stairs } \\
\text { steps and }> \\
50 \text { stairs steps. }\end{array}$ \\
\hline $\begin{array}{l}\text { van Landingham, } \\
\text { S. et al. (2012) [44] }\end{array}$ & $\begin{array}{l}\text { Age years: } 40+ \\
\text { Female: } 53 \%\end{array}$ & $\begin{array}{c}\text { Normal VF: } \\
n=1321 \\
\text { Unilateral VF } \\
\text { loss: } n=88 \\
\text { Bilateral VF loss: } \\
n=59\end{array}$ & $\begin{array}{c}\text { Accelerometer } \\
\text { (Actigraph, LLC, Ft. } \\
\text { Walton Beach, FL) }\end{array}$ & $\begin{array}{l}\text { VF Humphrey Matrix FDT } \\
\text { 19-point suprathreshold } \\
\text { screening test (N-30-5). } \\
\text { VA post refraction: Nidek } \\
\text { Autorefractor/Keratometer } \\
\text { (model ARK-760A) }\end{array}$ & No. & $\begin{array}{l}\text { Multivariable negative } \\
\text { binomial models to } \\
\text { assess relationship } \\
\text { between VF and PA. } \\
\text { Multivariate analysis } \\
\text { was also used to test } \\
\text { association between } \\
\text { other vision and } \\
\text { sociodemographic } \\
\text { factors on PA. }\end{array}$ & USA & $\begin{array}{l}\text { Covariates included in } \\
\text { the multivariable } \\
\text { models were age, sex, } \\
\text { race/ethnicity, and } \\
\text { education. Medical } \\
\text { comorbidities included } \\
\text { in the multivariable } \\
\text { models were chronic } \\
\text { obstructive pulmonary } \\
\text { disease/asthma, } \\
\text { arthritis, diabetes, } \\
\text { congestive heart } \\
\text { failure, and stroke. }\end{array}$ & $\begin{array}{c}\text { In multivariable models } \\
\text { bilateral VF loss but not } \\
\text { unilateral VF loss was } \\
\text { associated with fewer } \\
\text { daily steps }(p<0.05) \text { and } \\
\text { less MVPA }(p<0.01) . \\
\text { Post-refraction VA was } \\
\text { associated with fewer } \\
15 \% \text { daily steps }(p=0.04) \\
\text { and } 36 \% \text { less MVPA } \\
(p=0.04) .\end{array}$ \\
\hline
\end{tabular}


Table 1. Cont.

\begin{tabular}{|c|c|c|c|c|c|c|c|c|c|}
\hline Reference & $\begin{array}{c}\text { Population } \\
\text { Characteristics: Age } \\
\text { (Years), Gender. }\end{array}$ & Population: $(n)$ & $\begin{array}{l}\text { Physical Activity } \\
\text { Measurement Tool }\end{array}$ & Vision Measurement Tool & $\begin{array}{l}\text { Specific Eye } \\
\text { Disease } \\
\text { Examined? }\end{array}$ & Statistical Test & Country & $\begin{array}{l}\text { Confounders } \\
\text { Controlled for }\end{array}$ & Main Findings \\
\hline Zult, T., (2020) [45] & $\begin{array}{c}\text { AMD subjects with } \\
\text { vision loss: } \\
\text { Age (SD): } 76 \text { (7) } \\
\text { Male: } 45.5 \% \\
\text { Female: } 54.5 \% \\
\text { AMD subjects } \\
\text { without vision loss: } \\
\text { Age: } 76(7) \\
\text { Male: } 60 \% \\
\text { Female: } 50 \% \\
\text { Controls with } \\
\text { normal vision: } \\
\text { Age: } 70(4) \\
\text { Male: } 45.5 \% \\
\text { Female: } 54.5 \%\end{array}$ & $\begin{array}{c}\text { AMD subjects } \\
\text { with vision loss: } \\
n=11 \\
\text { AMD subjects } \\
\text { without vision } \\
\text { loss: } n=10 \\
\text { Control group } \\
\text { with normal } \\
\text { vision: } n=11\end{array}$ & $\begin{array}{l}\text { Accelerometer } \\
\text { (Actigraph GT3X } \\
\text { tri-axial), activity } \\
\text { monitor log and } \\
\text { interview using the } \\
\text { World Health } \\
\text { Organisation: Global } \\
\text { Physical Activity } \\
\text { Questionnaire. }\end{array}$ & $\begin{array}{l}\text { VA: Bailey-Lovie logMAR } \\
\text { chart } \\
\text { CS: Pelli-Robson chart } \\
\text { VF: Humphrey Field Analyzer } \\
\text { (Carl Zeiss Meditec Inc., } \\
\text { Dublin, CA, USA) } \\
\text { SITA-Standard } \\
\text { 30-2 threshold test }\end{array}$ & AMD & $\begin{array}{l}\text { Pearson's correlation } \\
\text { coefficients were } \\
\text { calculated to assess } \\
\text { whether there is a } \\
\text { relationship between } \\
\text { the severity of vision } \\
\text { loss and outcomes of } \\
\text { the Actigraph } \\
\text { and GPAQ. }\end{array}$ & $\begin{array}{c}\text { UK } \\
\text { (England) }\end{array}$ & $\begin{array}{l}\text { Control group was age } \\
\text { matched with AMD } \\
\text { group. }\end{array}$ & $\begin{array}{l}\text { There was a significant } \\
\text { negative correlation } \\
\text { between objectively } \\
\text { measured MVPA and } \\
\text { worse VA, VF and CS. } \\
\text { However, when } \\
\text { self-reported MVPA and } \\
\text { step count was examined, } \\
\text { the associations were } \\
\text { weaker and in the } \\
\text { opposite direction, } \\
\text { compared to when PA } \\
\text { levels were } \\
\text { objectively measured. }\end{array}$ \\
\hline
\end{tabular}

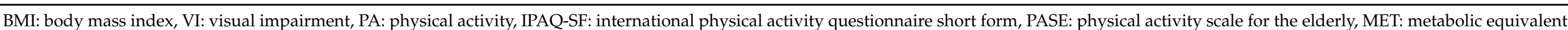

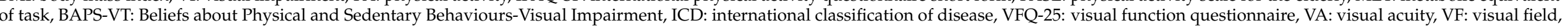

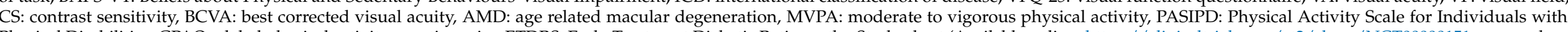

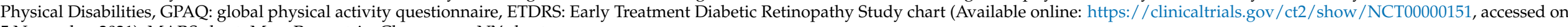
5 November 2021), MARS chart: Mars Perceptrix, Chappaqua, NY chart. 
Table 2. Quality assessment.

\begin{tabular}{ccc}
\hline High Quality & Medium Quality & Low Quality \\
\hline & Barnett, A. et al. [18] & \\
& Black, A. et al. [19] & Haegele, J. et al. (2016) [20] \\
& Haegele, J. et al. (2017) [23] & Haegele, J. et al. (2017) [21] \\
& Haegele, J. et al. (2021) [26] & Haegele, J. et al. (2017) [22] \\
& Inoue, S. et al. [29] & Haegele, J. et al. (2018) [24] \\
Loprinzi, S. et al. [34] & Jaarsma, E. et al. [30] & Haegele, J. et al. (2019) [25] \\
Shakarchi, A. et al. [41] & Jones, G. et al. [31] & Holbrook, E. et al. (2009) [27] \\
Ramulu, P. et al. (2019) [39] & Marmeleira, J. et al. (2014) [35] & Holbrook, K. et al. (2013) [28] \\
van Landingham, S. et al. & McMullan, I. et al. (2020) [7] & Labudzki, T. et al. (2013) [32] \\
(2012) [44] & Montarzino, A. et al. (2007) [36] & Lee M. et al. (2014) [33] \\
& Nguyen, A et al. (2015) [37] & Starkoff, B. et al. (2017) [42] \\
& Ramulu, P. et al. (2012) [38] & Zult, T., (2020) [45] \\
& Sengupta, N. et al. (2015) [40] & \\
\hline
\end{tabular}

\subsection{Non-Modifiable Correlates}

\subsubsection{Measures of Vision}

A range of different vision parameters and their association with MVPA were examined (Table 3), including self-reported VI classification (5/29), visual acuity in the better eye $(3 / 29)$, visual field (5/29), contrast sensitivity $(5 / 29)$ and colour vision $(1 / 29)$. For studies which examined the association between self-reported VI classification and MVPA, there was evidence that being classified as blind was associated with lower levels of PA when compared to being classified as VI [21,31]. However, there was no evidence across studies of a dose-response interaction between the severity of sight loss, based on the international blind sports classification of VI (B1 vs. B2 vs. B3 vs. B4), and MVPA [24,27,42]. All of the studies which examined the relationship between MVPA and VI classification relied on self-reported MVPA measurement tools. In addition, it is possible that three of the studies may have selected the same participants for multiple studies, due to the similarities in the methods used in the recruitment of participants [21,24,25].

Table 3. Measures of vision and association with MVPA.

\begin{tabular}{|c|c|c|c|c|c|c|}
\hline \multirow{2}{*}{$\begin{array}{c}\text { Measure of Vision } \\
\text { Self-Reported }\end{array}$} & \multicolumn{2}{|c|}{ Positive Association } & \multicolumn{2}{|c|}{ Negative Association } & \multicolumn{2}{|c|}{ No Association } \\
\hline & SR. PA & Obj.PA & SR. PA & Obj. PA & SR. PA & Obj. PA \\
\hline Self-reported VI classification (blindness vs VI) & & & {$[21,31]$} & & [42] & \\
\hline Self report VI classification (B1, B2, B3,B4) & & & & & {$[20,24,42]$} & \\
\hline Onset of VI (congenital vs after birth) & & & & & {$[20,22]$} & [35] \\
\hline $\begin{array}{l}\text { PA has an accumulative effect on PA over time via its } \\
\text { effect on vision }\end{array}$ & [36] & & & & & \\
\hline Years of VI & & & & & [20] & [35] \\
\hline Self-rated vision & & & & & [36] & \\
\hline \multirow[t]{2}{*}{ Objective Measures } & Positive & sociation & \multicolumn{2}{|c|}{ Negative Association } & \multicolumn{2}{|c|}{ No Association } \\
\hline & SR. PA & Obj. PA & SR. PA & Obj. PA & SR. PA & Obj. PA \\
\hline Contrast sensitivity (worse) & & & [19] & {$[40,45]$} & [45] & {$[37,38]$} \\
\hline Colour vision & & & & & & [41] \\
\hline Visual acuity without noise & & & & & & [41] \\
\hline Visual acuity (best seeing eye) (worse) & & & & {$[40,45]$} & {$[29,43,45]$} & \\
\hline VA (worse seeing eye) (worse) & & & [29] & & [43] & \\
\hline Visual field (worse) & & & & {$[37,38,44$,} & {$[19,45]$} & [44] \\
\hline Glaucoma (present) & & & & & & [38] \\
\hline Severe Glaucoma (present) & & & & [38] & & \\
\hline Stage of AMD & & & & & [43] & \\
\hline AMD present & & & & [40] & & \\
\hline Significant cataracts/PCO (present) & & & & [40] & & \\
\hline
\end{tabular}


In contrast, among studies which used objective measures of sight loss and of MVPA, there was stronger evidence from one high-quality study, two medium-quality studies and one low-quality study that visual field loss in the better eye, or in both eyes, was significantly associated with lower levels of MVPA [37,38,44,45] However, unilateral visual field loss was not associated with MVPA [44]. Another study used a principal component analysis with varimax rotation to establish three independent factors; the first factor, which loaded on to superior visual field measures, and the second factor, which loaded on to inferior visual field measures, were also not associated with self-reported MVPA [19]. One study reported that the binocular visual field was associated with MVPA when MVPA was objectively measured, but not when MVPA was self-reported in the same group of participants [45].

The most frequently studied vision parameters that were associated with walking were visual acuity in the better eye (3/29), visual field (3/29) and contrast sensitivity (3/29) (Table 4). Worse measures of visual field in the better eye [38], bilateral visual field [44] and integrated visual field (IVF) sensitivity, which was defined as a summary of the average overall and inferior field sensitivities [39], with a lower IVF representing a worse visual field, were associated with lower levels of walking.

Table 4. Measures of vision and association with walking.

\begin{tabular}{|c|c|c|c|c|c|c|}
\hline \multirow{2}{*}{$\begin{array}{c}\text { Measure of Vision } \\
\text { Self-Reported }\end{array}$} & \multicolumn{2}{|c|}{ Positive Association } & \multicolumn{2}{|c|}{ Negative Association } & \multicolumn{2}{|c|}{ No Association } \\
\hline & SR. PA & Obj. PA & SR. PA & Obj.PA & SR.PA & Obj.PA \\
\hline $\begin{array}{l}\text { Vision loss (self-reported } \\
\text { blindness vs. self-reported VI) }\end{array}$ & & & {$[42]^{*}$} & & & \\
\hline Self-report VI classification & & & {$[42]^{* *}$} & & & \\
\hline $\begin{array}{l}\text { Onset of VI (Congenital vs. } \\
\text { acquired blindness) }\end{array}$ & & & & & & [35] \\
\hline Years of VI/Age of onset & & & & & & [35] \\
\hline Severity of VI $\times$ Gender & & & & & & [25] \\
\hline \multirow[t]{2}{*}{ Objective Measures } & \multicolumn{2}{|c|}{ Positive Association } & \multicolumn{2}{|c|}{ Negative Association } & \multicolumn{2}{|c|}{ No Association } \\
\hline & SR. PA & Obj.PA & SR. PA & Obj. PA & SR. PA & Obj. PA \\
\hline Contrast sensitivity (worse) & & & & & & {$[38,40,45]$} \\
\hline $\begin{array}{l}\text { Visual acuity (Best seeing } \\
\text { eye) (worse) }\end{array}$ & & & $\begin{array}{c}{[36]^{* * *}} \\
\text { [43] (stairs } \\
\text { taken daily) }\end{array}$ & [40] & {$[36,43], * * *$} & \\
\hline Visual acuity (Worse seeing eye) & & & $\begin{array}{l}\text { [43] (stairs } \\
\text { taken daily) }\end{array}$ & & [43] & [44] \\
\hline Visual field (worse) & & & & $\begin{array}{c}\text { [39] (Integrated } \\
\text { visual field } \\
\text { sensitivity) } \\
\text { [38] (visual field } \\
\text { loss in better eye) } \\
\text { [45] }\end{array}$ & & $\begin{array}{c}\text { [45] (unilateral } \\
\text { visual field loss was } \\
\text { not associated with } \\
\text { less steps) } \\
\text { [44] }\end{array}$ \\
\hline Vision parameters & & & & & & [41] \\
\hline Glaucoma (present) & & & & & & [38] \\
\hline Glaucoma (severe) (present) & & & & [38] & & \\
\hline AMD (present) & & [40] & & & & \\
\hline Stage of AMD & & & & & [43] & \\
\hline Sig. Cataract/PCO & & & & & & [40] \\
\hline
\end{tabular}

* Participants classified as B2 spent significantly more minutes of walking than participants classified as B1. ** Strakoff, B.E. (2017) reported a significant difference in walking between participants who self-reported VI categories B1 vs. B2 vs. B3 vs. B4. The mean min/day of participants classified as B1 was $46.8 \mathrm{~min}$, com-pared to $95.8 \mathrm{~min}$ for participants in B2. However, there was no dose response identified as participants in the B3 category engaged in a mean of 62.6 min per day of walking. ${ }^{* * *}$ In regression analysis self-reported VA diagnosis, was a discriminating factor in walking for those over the age of 77 with a breaking point for those under and over 87.5 years. 
Studies which explored the association between visual acuity in the better eye and walking were all considered medium-quality studies and reported mixed results. One study, which used objective measures of walking, reported a significant negative relationship between visual acuity and daily steps taken [40]. In contrast, two studies which examined walking reported different outcomes which were dependent on the nature of the walking, or the group, examined. One study that reported walking was not associated with visual acuity in the better eye when the weekly time walked was examined, however, there was a negative association between visual acuity in the better eye and daily stairs taken [43]. Another study reported that the self-reported visual acuity status was a discriminating factor in walking distance for those over the age of 77, with a breaking point for those under and over 87.5 years [36].

The studies in the review reported mixed results for the association between PA and contrast sensitivity. Worse contrast sensitivity was found to be associated with lower levels of objectively measured MVPA in two studies, [40,45] and self-reported MVPA in one study [19]. However, contrast sensitivity was also found to have no association with objectively measured MVPA in two studies [37,38], and self-reported MVPA in one study [45]. For walking, there was no significant association found in any of the three studies between contrast sensitivity and objectively measured walking [38,40,45].

\subsubsection{Personal Correlates}

In terms of the variables classified as non-modifiable personal correlates, twelve variables were tested for their association with MVPA (Table 5), and six variables were tested for their association with walking (Table 6). The most frequently reported correlation was between PA and gender. Overall, three studies reported that male participants selfreported engaging in significantly more MVPA than female participants [23,27,42], and five studies reported that there was no association between self-reported MVPA and gender $[21,22,24,29,32]$. One low-quality study examined the relationship between objectively measured MVPA and gender and reported no association between gender and MVPA [35]. For walking, two low-quality studies, one that relied on self-reported walking and the other that relied on objectively measured walking, both reported no association between walking and gender [35,42].

Table 5. Personal non-modifiable variables and their association with MVPA.

\begin{tabular}{|c|c|c|c|c|c|c|}
\hline \multirow[t]{2}{*}{ Personal Characteristics } & \multicolumn{2}{|c|}{ Positive Association } & \multicolumn{2}{|c|}{ Negative Association } & \multicolumn{2}{|c|}{ No Association } \\
\hline & SR. PA & Obj. PA & SR. PA & Obj. PA & SR. PA & Obj. PA \\
\hline Comorbidities & & & & & [29] & \\
\hline Hearing loss (dual sensory impairment) & & & & [34] & & \\
\hline BMI (Higher) & & & {$[26,33]$} & & {$[24,29,42]$} & [35] \\
\hline Use of a mobility aid & & & & & [20] & \\
\hline Health related quality of life (higher) & [23] & & & & & \\
\hline Depression & & & & & [26] & \\
\hline Level of independence (Higher) & [23] & & & & & \\
\hline Age (older) & & & & & {$[21,22,24,26,29]$} & \\
\hline Gender (men) & {$[20,23,42]$} & & & & {$[21,22,24,29,31]$} & [35] \\
\hline Household income & & & & & [24] & \\
\hline Ethnicity- (comparing Caucasian, African & & & & & & \\
\hline $\begin{array}{l}\text { America, Asian, Hispanics and other } \\
\text { ethnic groups) }\end{array}$ & & & & & [20] & \\
\hline Education & & & & & [20] & \\
\hline
\end{tabular}


Table 6. Personal non-modifiable variables and their association with walking.

\begin{tabular}{|c|c|c|c|c|c|c|}
\hline \multirow[t]{2}{*}{ Personal } & \multicolumn{2}{|c|}{ Positive Association } & \multicolumn{2}{|c|}{ Negative Association } & \multicolumn{2}{|c|}{ No Association } \\
\hline & SR. PA & Obj. PA & SR. PA & Obj. PA & SR.PA & Obj.PA \\
\hline Comorbidities & & & & & & [39] \\
\hline Polypharmacy ( $\geq 5$ vs. $<5$ non-eye drop medication) & & & & [39] & & \\
\hline BMI (Higher) & & & & & [42] & [35] \\
\hline Gender (male) & & & & & [42] & [35] \\
\hline Severity of VI $\times$ Gender & & & & & & [25] \\
\hline Age (older) & & & {$[36]^{* *}$} & & {$[36]^{* *}$} & \\
\hline
\end{tabular}

** The influence of age on walking was dependent on the sub-group examined. Among younger participants, with worse vision and who were more active, age was reported to have a greater impact on walking than vision loss.

Older age was not found to be associated with self-reported MVPA in any of the four studies which examined MVPA [21,22,24,29]. One study reported that older age was associated with participants who self-reported less walking, however, the influence of age on walking was dependent on the sub-group examined. Among younger participants with poorer sight and who were more active, age was reported to have a greater impact on walking than sight loss [36]. No studies used objective measures of MVPA or walking to examine the association between PA and age.

Comorbidities, BMI, health related quality of life, depression, and level of independence were also included as non-modifiable personal correlates. These factors could have a bidirectional relationship with PA, which could also make them modifiable correlates. For example, improvements in depressive symptoms could help an individual feel more energised, and thus they are more likely to engage in PA.

\subsection{Modifiable}

\subsubsection{Personal Correlates}

A range of psychosocial factors and their association with MVPA were examined, including self-efficacy [24,25] social support [21], self-regulation [21], the perceived barriers to PA [33], the theory of planned behaviour constructs (attitude towards PA, subjective norm, perceived behaviour control, intention to engage in PA) [22], use of a mobility aid [20], and levels of self-reported independence [23] (Table 7). Whilst only two studies, which were both conducted in similar cohorts, explored the relationship between MVPA and self-efficacy, the results from other studies provided evidence of factors which may also influence self-efficacy, defined as 'the belief an individual has in their ability to perform a task and to obtain the desired results'. For example, a fear of falling was found to mediate the relationship between sight loss and PA in one study that was included in our review [37]. Perceived barriers to PA [33], and a lower self-reported level of independence [23] also had a negative and significant association with MVPA; it is plausible that these factors would influence an individual's self-efficacy for PA. Social support was also reported to be positively associated with MVPA in one low-quality study [21], and peer/buddy support was also found to have a significant positive association with sports participation in a medium-quality study [30]. No studies explored psychosocial factors that were associated with walking. 
Table 7. Modifiable personal correlates of MVPA.

\begin{tabular}{|c|c|c|c|c|c|c|}
\hline \multirow[t]{2}{*}{ Personal Factors } & \multicolumn{2}{|c|}{ Positive Association } & \multicolumn{2}{|c|}{ Negative Association } & \multicolumn{2}{|c|}{ No Association } \\
\hline & SR. PA & Obj. PA & SR. PA & Obj. PA & SR. PA & Obj. PA \\
\hline Self efficacy & {$[24,25]$} & & & & & \\
\hline Self-regulation & & & & & [21] & \\
\hline Social support & [21] & & & & & \\
\hline Intention to engage in PA & [22] & & & & & \\
\hline $\begin{array}{l}\text { Attitudes/beliefs (theory of planned } \\
\text { behaviour constructs) }\end{array}$ & & & & & [22] & \\
\hline Sedentary behaviour (more time in SB) & & & [23] & & {$[22,26]$} & \\
\hline Level of independence (Higher) & [23] & & & & & \\
\hline Use of mobility aid & & & & & [20] & \\
\hline Fewer perceived PA barriers & [33] & & & & & \\
\hline Sleep time (Higher) & & & & & [26] & \\
\hline
\end{tabular}

\subsubsection{Environmental Correlates}

When compared to the non-modifiable factors that were associated with MVPA, the modifiable environmental factors were less researched (Table 8). One low-quality study reported a negative correlation coefficient between the logit of the perceived barriers to PA and self-reported PA; included among the most severe barriers to PA which were cited by participants was the environmental barrier: 'lack of transportation to get to places to exercise' [33]. One medium-quality study found a positive association between access to services and non-walking PA, and a negative association between physical barriers to walking and non-walking PA [18].

Table 8. Environmental factors associated with MVPA.

\begin{tabular}{|c|c|c|c|c|c|c|}
\hline \multirow[t]{2}{*}{ Environmental } & \multicolumn{2}{|c|}{ Positive Association } & \multicolumn{2}{|c|}{ Negative Association } & \multicolumn{2}{|c|}{ No Association } \\
\hline & SR. PA & Obj. PA & SR. PA & Obj. PA & SR. PA & Obj. PA \\
\hline Fewer perceived PA barriers & [33] & & & & & \\
\hline Land use mix- access to services ( 1 unit increase) & [18] & & & & & \\
\hline Physical barriers to walking (1 unit increase) & & & [18] & & & \\
\hline
\end{tabular}

Three studies examined environmental variables and their association with walking (Table 9). There was evidence from one medium-quality study, which used self-reported measures of walking, that the number of years lived at the same address was associated with increased walking, whilst feeling unsafe while walking around the neighbourhood was associated with less walking [36]. Another medium-quality study, which relied on selfreported walking measures, found no association between walking and neighbourhood aesthetics [18]. Only one low-quality study used objective measures of walking and found that there was no association between day of the week and walking [28].

Table 9. Environmental Factors associated with walking.

\begin{tabular}{|c|c|c|c|c|c|c|}
\hline \multirow[t]{2}{*}{ Environmental } & \multicolumn{2}{|c|}{$\begin{array}{c}\text { Positive } \\
\text { Association }\end{array}$} & \multicolumn{2}{|c|}{$\begin{array}{c}\text { Negative } \\
\text { Association }\end{array}$} & \multicolumn{2}{|c|}{ No Association } \\
\hline & SR. PA & Obj. PA & SR.PA & Obj.PA & SR.PA & Obj.PA \\
\hline Feeling of safety when walking in the neighbourhood (worse) & & & [36] & & & \\
\hline Years lived at the same address (i.e., neighbourhood familiarity) & [36] & & & & & \\
\hline Neighbourhood aesthetics & & & & & {$[18]$} & \\
\hline
\end{tabular}

Due to the variations between PA measurement tools and study design, it was not appropriate to conduct a meta-analysis for this review. 


\section{Discussion}

Our review aimed to identify modifiable and non-modifiable correlates of PA among people with sight loss. Evidence from multiple studies reported that the VI category, worse visual acuity, bilateral visual field loss, worse contrast sensitivity, individuals of female gender, lower self-efficacy, and environmental barriers were associated with lower levels of PA among populations with sight loss.

Visual field and visual acuity are common measurements taken during a routine eye examination that is carried out by an optometrist or ophthalmologist. The measures of visual field and visual acuity are also used to classify people as blind or vision impaired. Our findings that worse visual acuity, bilateral visual field loss, and being classified as blind versus visually impaired could be associated with lower PA highlight that optometrists or ophthalmologists may be important for identifying populations at risk of low PA. Optometrists and ophthalmologists could therefore work with community groups and low vision services, to refer populations identified as being at risk of low PA to PA opportunities and support services. However, the vision parameters did not fully explain the variances in PA. Our review found additional non-modifiable and modifiable correlates of PA, which may have important implications for future PA interventions.

In terms of non-modifiable personal factors which correlate with PA, we found evidence that male participants were more likely to engage in higher levels of PA than female participants. These findings are in line with previous research in sighted populations. Globally, and particularly in HIC Western countries, men are, on average, more physically active than women [47]. However, one study in our review found that the relationship between gender and PA was mediated by social support and self-regulation [21]. Although these findings are limited to one study, which had a small sample size, the results highlight the importance of understanding the mechanisms which may lead to gender differences in PA. An intervention aimed at targeting low PA among women may not be effective if it does not target the mechanisms which result in women engaging in less PA than men.

Mixed results were found regarding the relationship between age and PA among people with sight loss. In sighted populations, research has consistently reported lower levels of MVPA that are associated with older age [48]. It is possible that because sight loss is associated with older age, the studies did not have a large enough sample of young adults with sight loss to be able to identify a negative association between older age and PA levels. Given that all age groups were identified as being at a possible risk of low PA, the findings highlight that multiple age groups could benefit from being targeted in PA interventions.

Importantly, our review also highlighted several areas where there is a lack of research. Firstly, there was limited research that explored the association between ethnicity and PA levels among people with sight loss. Among sighted populations, there is a large body of evidence that PA varies between ethnic groups within the UK $[49,50]$ It is important to understand the PA differences between ethnic groups, to ensure that PA interventions do not compound the existing ethnic inequalities regarding the access to sight loss services [51]. In addition, there was a lack of research that explored the association between additional disabilities, as well as sight loss, and PA. Research suggests that additional disabilities are common among people with sight loss. For example, one in three people in the UK with a learning disability is estimated to be affected by a sight problem [52]. It is plausible that having multiple disabilities, including sight loss, may limit an individual's opportunities and ability to engage in PA, thus increasing that individual's risk of low PA. Therefore, it is important that interventions can be adapted to accommodate for people with additional disabilities, as well as sight loss. Further to this, there was a lack of research that explored the association between mobility measures and PA levels in populations with sight loss. Mobility measures may be modifiable by PA interventions, as research suggests PA programs could attenuate differences in gait and functional parameters between populations with sight loss and sighted populations [53]. Improvements in mobility among VI populations could also improve mental health outcomes, as previous research has found 
gait speed to be a significant predictor of depressive symptoms in VI populations [54]. Therefore, there is a need to understand the bidirectional relationship between PA and mobility measures within populations with sight loss, to determine how mobility measures should be targeted in an intervention to increase PA, and to determine target population groups (e.g., individuals with a slower gait speed).

The review also identified a range of psychosocial factors that are associated with low PA, including lower levels of social support, self-efficacy, intention to engage in PA, perceived barriers to PA, and lower levels of self-reported independence. There are a range of interventions which could be used to target these psychosocial factors; for example, group-based PA may encourage social support, whilst PA that is prescribed by a health professional could promote an intention to engage in PA. In addition, sight loss services may be able to support people in becoming more independent, and reduce the impact of barriers to PA, by supporting people in maximising their residual vision and improve daily functioning.

When compared to non-modifiable variables that were associated with PA, modifiable factors that were associated with the environment were less researched. Our review reported that an access to services, physical barriers, fears of safety, and perceived barriers, including 'lack of transportation to get to places to exercise', as well as familiarity with the neighbourhood, were associated with lower PA among people with sight loss. These could be considered barriers to PA which could be addressed by sight loss services; for example, orientation and mobility training could reduce individuals' fears of safety in the neighbourhood. However, it is important that policies and planning also ensure that environments are designed to be accessible, and that the interventions to promote PA should consider environmental changes which can facilitate PA, as well as individual support to increase PA. Future research should also explore accessibility and mobility barriers which exist in low-income countries. It is plausible that the environmental barriers to PA that were identified in HIC countries in this review may be more pronounced in low-income countries, due to a lack of investment in public transport and infrastructure which make the streets more accessible, such as tactile paving, signal controlled pedestrian crossings, maintained pavements and detectable kerbs that separate traffic from pedestrians.

However, there were limitations to this review. Firstly, there were a limited number of studies which used objective measures of both sight loss and PA, which are considered more reliable and valid tools than self-reporting measurement tools. One study reported that in a group of patients with AMD, lower MVPA measured by objective tools was associated with worse visual acuity, visual field and contrast sensitivity, whereas when the same participants' self-reported measures of PA were examined, the study did not report a significant negative correlation [45]. Although this study included a small sample size, the findings indicated the influence that a measurement tool can have on the outcome results. In addition, sight loss was defined and measured using a range of methods, and the studies used different criteria to define visual impairment and blindness, thus limiting the comparability of results. Future research that is conducted among populations with sight loss should adopt a standard definition of sight loss, to ensure the future comparability between studies. We suggest that sight loss can be defined as a self-reported 'sight loss whilst wearing corrective devices', however, a follow-up, standardised eye test should be used, if available, to describe the degree and type of sight loss of the population being studied. In the absence of eye testing equipment or expertise, then follow-up questions should be asked to understand the nature of the participants sight loss. Although some studies objectively measured vision and PA using validated tools, these studies were often limited by a smaller sample size than those which relied on self-reported measures. There were also limitations among studies as a result of the recruitment procedures. Studies which recruited participants via online channels that were distributed by a VI organisation risked selection bias, which resulted in younger and more active participants than the overall population of people living with sight loss. In addition, people with additional disabilities, in particular, cognitive impairments such as dementia and learning difficulties, 
may be excluded from studies if they are unable to understand the PA survey or provide informed consent. Therefore, the correlates of PA may not be representative of the barriers experienced by populations with the lowest levels of PA.

\section{Conclusions}

Overall, our findings have highlighted the complexities of the factors which are associated with PA behaviour among people with sight loss in HICs. Optometrists and ophthalmologists are well positioned to identify patients with sight loss who may be at risk of low PA, and collaborate with sight loss services (e.g., charities, community groups, and council services) to refer people to PA advice and support. In addition, people working in the delivery of sight loss services may be able to support people by addressing the barriers to PA, and promoting greater independence, which could facilitate PA. However, PA is complex and our review highlighted the need for PA interventions to meet the needs of a range of population groups with sight loss. We suggest that future research aims to understand how different sectors and services could identify people at risk of low PA, and work together to provide individualised support to promote PA.

\section{Implications for Practice}

- Worse visual acuity and visual field may indicate that an individual is at risk of lower PA [29,36-38,40,43-45]. These measures are examined in routine eye tests, thus optometrists and ophthalmologists could identify people at risk of low PA, and play a key role in referring people to PA groups and opportunities.

- It is important to understand how factors including gender, age, ethnicity, and additional disabilities influence PA in the context in which interventions are being delivered. Interventions should work with communities to understand local needs, develop appropriate interventions, and target different sociodemographic groups, when appropriate $[55,56]$.

- Interventions should consider the environmental factors, such as unsafe streets [41], and a limited access to services [18] which influence PA and make adjustments to minimise these barriers.

- Future studies, with larger, representative sample sizes, and objectively measured PA, are required, to explore the findings in studies which are currently limited to a small evidence base.

Author Contributions: R.K.L., L.S. and P.M.A. were responsible for the manuscript conceptualization. R.K.L. prepared the original draft, and R.K.L., F.D.G., P.M.A., M.A.T., C.M., D.P., T.G., Y.B. and L.S. contributed to reviewing and editing. All authors have read and agreed to the published version of the manuscript.

Funding: This research received no external funding.

Institutional Review Board Statement: Not applicable.

Informed Consent Statement: Not applicable.

Data Availability Statement: Not applicable.

Conflicts of Interest: The authors declare no conflict of interest.

\section{Appendix A}

("activities of daily living" OR "physical activity" OR "lifestyle activity" OR "inactive" OR "insufficientactivity" OR "mobility" OR "incidental activity" OR "walking" OR "active transport" OR "non exercise activity thermogenesis" OR "lipa" OR "lpa" OR "neat" OR "light exercise" OR "moderate exercise" OR "nepa" OR "sport" OR “exercise" OR "walking") AND (Barrier* OR facilitator* OR modifier* OR motivator* OR influences OR uptake OR engagement OR correlate* OR encourage OR obstacle OR prevents OR participation OR predictor OR mediator OR moderator) AND ("Visually impaired" OR "Sight 
loss" OR "Sight impairment" OR "macular degeneration" OR AMD OR "Uncorrected refractive error" OR "Glaucoma" OR "Diabetes retinopathy" OR “Eye impairment" OR "Visual impairment" OR "Vision disorder *" OR "Vision impaired" OR "low vision" OR "ocular pathology").

\section{References}

1. Caspersen, C.J.; Powell, K.E.; Christenson, G.M. Physical activity, exercise, and physical fitness: Definitions and distinctions for health-related research. Public Health Rep. 1985, 100, 126-131.

2. Bull, F.C.; Al-Ansari, S.S.; Biddle, S.; Borodulin, K.; Buman, M.P.; Cardon, G.; Carty, C.; Chaput, J.-P.; Chastin, S.; Chou, R.; et al. World Health Organization 2020 guidelines on physical activity and sedentary behaviour. Br. J. Sports Med. 2020, 54, 1451-1462. [CrossRef] [PubMed]

3. Lee, I.; Shiroma, E.; Lobelo, F.; Puska, P.; Blair, S.; Katzmarzyk, P. Effect of physical inactivity on major non-communicable diseases worldwide: An analysis of burden of disease and life expectancy. Lancet 2012, 380, 219-229. [CrossRef]

4. World Health Organisation. World Report on Vision. 2019. Available online: https://www.who.int/publications/i/item/worldreport-on-vision (accessed on 22 October 2021).

5. World Health Organisation. Blindness and Vision Impairment. 2021. Available online: https://www.who.int/news-room/factsheets / detail/blindness-and-visual-impairment (accessed on 5 November 2021).

6. Royal National Institute of Blind People (RNIB). The Economic Impact of Sight Loss and Blindness in the UK Adult Population. 2013. Available online: www.deloitte.com/au/about (accessed on 22 October 2021).

7. McMullan, I.I.; Bunting, B.P.; Smith, L.; Koyanagi, A.; Tully, M.A. Is There an Association Between Self-Reported Physical Activity and Self-Rated Vision Over Time? Results From the Irish Longitudinal Study of Ageing. J. Aging Phys. Act. 2020, 28, 1-8. [CrossRef] [PubMed]

8. Willis, J.R.; Jefferys, J.L.; Vitale, S.; Ramulu, P.Y. Visual impairment, uncorrected refractive error, and accelerometer-defined physical activity in the United States. Arch. Ophthalmol. 2012, 130, 329-335. [CrossRef]

9. Smith, L.; A Timmis, M.; Pardhan, S.; Latham, K.; Johnstone, J.; Hamer, M. Physical inactivity in relation to self-rated eyesight: Cross-sectional analysis from the English Longitudinal Study of Ageing. BMJ Open Ophthalmol. 2017, 1, e000046. [CrossRef]

10. Brunes, A.; Flanders, W.D.; Augestad, L.B. Self-reported visual impairment, physical activity and all-cause mortality: The HUNT Study. Scand. J. Public Health 2016, 45, 33-41. [CrossRef]

11. Loprinzi, P.D.; Joyner, C. Accelerometer-determined physical activity and mortality in a national prospective cohort study: Considerations by visual acuity. Prev. Med. 2016, 87, 18-21. [CrossRef]

12. Kim, K.-N.; Park, S.J.; Kim, W.; Joo, J.; Kim, H.; Kim, K.H.; Sohn, J.H.; Kwon, Y.J. Modification of the Association between Visual Impairment and Mortality by Physical Activity: A Cohort Study among the Korean National Health Examinees. Int. J. Environ. Res. Public Health 2019, 16, 4386. [CrossRef]

13. Sweeting, J.; Merom, D.; Astuti, P.A.S.; Antoun, M.; Edwards, K.; Ding, D. Physical activity interventions for adults who are visually impaired: A systematic review and meta-analysis. BMJ Open 2020, 10, e034036. [CrossRef]

14. NHS. Age Related Macular Degeneration. Available online: https://www.nhs.uk/conditions/age-related-macular-degenerationamd/ (accessed on 5 November 2021).

15. National Eye Institute. Retinitis Pigmentosa. Available online: https://www.nei.nih.gov/learn-about-eye-health/eye-conditionsand-diseases / retinitis-pigmentosa (accessed on 5 November 2021).

16. Page, M.J.; McKenzie, J.E.; Bossuyt, P.M.; Boutron, I.; Hoffmann, T.C.; Mulrow, C.D.; Shamseer, L.; Tetzlaff, J.M.; Akl, E.A.; Brennan, S.E.; et al. The PRISMA 2020 statement: An updated guideline for reporting systematic reviews. BMJ 2021, 372, n71. [CrossRef] [PubMed]

17. Li, Q.D.; Kuang, X.M.; Qi, J. Correlates of Physical Activity of Children and Adolescents with Visual Impairments: A Systematic Review. Curr. Pharm. Des. 2020, 26, 5002-5011. [CrossRef] [PubMed]

18. Barnett, A.; Cerin, E.; Zhang, C.J.P.; Sit, C.H.P.; Johnston, J.M.; Cheung, M.M.C.; Lee, R.S.Y. Associations between the neighbourhood environment characteristics and physical activity in older adults with specific types of chronic conditions: The ALECS cross-sectional study. Int. J. Behav. Nutr. Phys. Act. 2016, 13, 1-13. [CrossRef]

19. Black, A.A.; Wood, J.M.; Lovie-Kitchin, J.E. Inferior visual field reductions are associated with poorer functional status among older adults with glaucoma. Ophthalmic Physiol. Opt. 2011, 31, 283-291. [CrossRef]

20. Haegele, J.A.; Zhu, X.; Lee, J.; Lieberman, L.J. Physical Activity for Adults with Visual Impairments: Impact of Socio-Demographic Factors. Eur. J. Adapt. Phys. Act. 2016, 9, 3-14. [CrossRef]

21. Haegele, J.A.; Brian, A.S.; Lieberman, L.J. Social Cognitive Theory Determinants of Physical Activity in Adults with Visual Impairments. J. Dev. Phys. Disabil. 2017, 29, 911-923. [CrossRef]

22. Haegele, J.A.; Hodge, S.R.; Kozub, F.M. Beliefs about physical activity and sedentary behaviors of adults with visual impairments. Disabil. Health J. 2017, 10, 571-579. [CrossRef]

23. Haegele, J.A.; Famelia, R.; Lee, J. Health-related quality of life, physical activity, and sedentary behavior of adults with visual impairments. Disabil. Rehabil. 2016, 39, 2269-2276. [CrossRef]

24. Haegele, J.A.; Kirk, T.N.; Zhu, X. Self-efficacy and physical activity among adults with visual impairments. Disabil. Health J. 2018, 11, 324-329. [CrossRef] [PubMed] 
25. Haegele, J.A.; Zhu, X. Physical Activity, Self-efficacy and Health-related Quality of Life among Adults with Visual Impairments. Disabil. Rehabil. 2019, 43, 530-536. [CrossRef] [PubMed]

26. Haegele, J.A.; Zhu, X.; Healy, S. Behavioral Correlates of Depression Among Adults with Visual Impairments. J. Vis. Impair. Blind. 2021, 115, 403-413. [CrossRef]

27. Holbrook, E.A.; Caputo, J.L.; Perry, T.L.; Fuller, D.K.; Morgans, D.W. Physical activity, body composition, and perceived quality of life of adults with visual impairments. J. Vis. Impair. Blind. 2009, 103, 17-29. [CrossRef]

28. Holbrook, E.A.; Kang, M.; Morgan, N.W. Acquiring a stable estimate of physical activity in adults with visual impairment. Adapt. Phys. Act. Q. 2013, 30, 59-69. [CrossRef] [PubMed]

29. Inoue, S.; Kawashima, M.; Hiratsuka, Y.; Nakano, T.; Tamura, H.; Ono, K.; Mrukami, A.; Tsubota, K.; Yamada, M. Assessment of physical inactivity and locomotor dysfunction in adults with visual impairment. Sci. Rep. 2018, 8, 12032. [CrossRef] [PubMed]

30. Jaarsma, E.A.; Dekker, R.; Koopmans, S.A.; Dijkstra, P.U.; Geertzen, J.H.B. Barriers to and Facilitators of Sports Participation in People with Visual Impairments. Adapt. Phys. Act. Q. 2014, 31, 240-264. [CrossRef]

31. Jones, G.C.; Crews, J.E.; Danielson, M.L. Health risk profile for older adults with blindness: An application of the international classification of functioning, disability, and health framework. Ophthalmic Epidemiol. 2010, 17, 400-410. [CrossRef]

32. Łabudzki, J.; Tasiemski, T. Physical activity and life satisfaction in blind and visually impaired individuals. Hum. Mov. 2013, 14, 210-216. [CrossRef]

33. Lee, M.; Zhu, W.; Ackley-Holbrook, E.; Brower, D.G.; McMurray, B. Calibration and validation of the Physical Activity Barrier Scale for persons who are blind or visually impaired. Disabil. Health J. 2014, 7, 309-317. [CrossRef]

34. Loprinzi, P.D.; Smit, E.; Lin, F.R.; Gilham, B.; Ramulu, P.Y. Accelerometer-assessed physical activity and objectively determined dual sensory impairment in US adults. Mayo Clin. Proc. 2013, 88, 690-696. [CrossRef]

35. Marmeleira, J.; Laranjo, L.; Marques, O.; Pereira, C. Physical activity patterns in adults who are blind as assessed by accelerometry. Adapt. Phys. Act. Q. 2014, 31, 283-296. [CrossRef]

36. Montarzino, A.; Robertson, B.; Aspinall, P.; Ambrecht, A.; Findlay, C.; Hine, J.; Dhillon, B. The Impact of Mobility and Public Transport on the Independence of Visually Impaired People. Vis. Impair. Res. 2007, 9, 67-82. [CrossRef]

37. Nguyen, A.M.; Arora, K.S.; Swenor, B.K.; Friedman, D.S.; Ramulu, P.Y. Physical activity restriction in age-related eye disease: A cross-sectional study exploring fear of falling as a potential mediator Physical functioning, physical health and activity. BMC Geriatr. 2015, 15, 1-10. [CrossRef]

38. Ramulu, P.Y.; Maul, E.; Hochberg, C.; Chan, E.S.; Ferrucci, L.; Friedman, D. Real-World Assessment of Physical Activity in Glaucoma Using an Accelerometer. Ophthalmology 2012, 119, 1159-1166. [CrossRef]

39. Ramulu, P.Y.; Ms, A.M.; West, S.K.; Friedman, D.S.; Gitlin, L.N. What Is a Falls Risk Factor? Factors Associated with Falls per Time or per Step in Individuals with Glaucoma. J. Am. Geriatr. Soc. 2018, 67, 87-92. [CrossRef]

40. Sengupta, S.; Nguyen, A.M.; van Landingham, S.W.; Solomon, S.D.; Do, D.V.; Ferrucci, L.; Friedman, D.S.; Ramulu, P.Y. Evaluation of real-world mobility in age-related macular degeneration. BMC Ophthalmol. 2015, 15, 9. [CrossRef] [PubMed]

41. Shakarchi, A.F.; Mihailovic, A.; West, S.K.; Friedman, D.; Ramulu, P.Y. Vision Parameters Most Important to Functionality in Glaucoma. Investig. Opthalmology Vis. Sci. 2019, 60, 4556-4563. [CrossRef]

42. Starkoff, B.E.; Lenz, E.K.; Lieberman, L.J.; Foley, J.; Too, D. Physical activity patterns of adults with visual impairments. Br. J. Vis. Impair. 2017, 35, 130-142. [CrossRef]

43. Subhi, Y.; Sørensen, T.L. Physical activity patterns in patients with early and late age-related macular degeneration. Dan. Med. J. 2016, 63, A5303.

44. van Landingham, S.W.; Willis, J.R.; Vitale, S.; Ramulu, P.Y. Visual field loss and accelerometer-measured physical activity in the United States. Ophthalmology 2012, 119, 2486-2492. [CrossRef]

45. Zult, T.; Smith, L.; Stringer, C.; Pardhan, S. Levels of self-reported and objective physical activity in individuals with age-related macular degeneration. BMC Public Health 2020, 20, 1144. [CrossRef] [PubMed]

46. Critical Appraisal Program. CASP Cohort Study Checklist. 2018. Available online: https://casp-uk.net/wp-content/uploads/20 18/03/CASP-Cohort-Study-Checklist-2018_fillable_form.pdf (accessed on 4 May 2021).

47. Guthold, R.; A Stevens, G.; Riley, L.M.; Bull, F.C. Worldwide trends in insufficient physical activity from 2001 to 2016 : A pooled analysis of 358 population-based surveys with 1.9 million participants. Lancet Glob. Health 2018, 6, e1077-e1086. [CrossRef]

48. Sallis, J.F. Age-related decline in physical activity: A synthesis of human and animal studies. Med. Sci. Sports Exerc. 2000, 32, 1598-1600. [CrossRef]

49. GOV.UK. Physical Activity. 2020. Available online: https://www.ethnicity-facts-figures.service.gov.uk/health/diet-andexercise/ physical-activity/latest\#by-ethnicity-over-time (accessed on 4 June 2021).

50. Bhatnagar, P.; Townsend, N.; Shaw, A.; Foster, C. The physical activity profiles of South Asian ethnic groups in England. J. Epidemiol. Community Health 2015, 70, 602-608. [CrossRef]

51. Johnson, M.R.; Morjaria-Keval, A. Ethnicity, sight loss and invisibility. Br. J. Vis. Impair. 2007, 25, 21-31. [CrossRef]

52. RNIB. Learning Disability and Sight Loss 2013. Available online: http://www.rnib.org.uk/learningdisability (accessed on 5 November 2021).

53. da Silva, E.S.; Fischer, G.; da Rosa, R.G.; Schons, P.; Teixeira, L.B.T.; Hoogkamer, W.; Peyré-Tartaruga, L.A. Gait and functionality of individuals with visual impairment who participate in sports. Gait Posture 2018, 62, 355-358. [CrossRef] 
54. Gleeson, M.; Sherrington, C.; Lo, S.; Auld, R.; Keay, L. Impact of the Alexander technique on well-being: A randomised controlled trial involving older adults with visual impairment. Clin. Exp. Optom. 2017, 100, 633-641. [CrossRef] [PubMed]

55. Moore, G.F.; Evans, R.E.; Hawkins, J.; Littlecott, H.; Melendez-Torres, G.; Bonell, C.; Murphy, S. From complex social interventions to interventions in complex social systems: Future directions and unresolved questions for intervention development and evaluation. Evaluation 2018, 25, 23-45. [CrossRef] [PubMed]

56. Craig, P.; Di Ruggiero, E.; Frohlich, K.L.; Mykhalovskiy, E.; White, M.; Campbell, R.; Cummins, S.; Edwards, N.; Hunt, K.; Kee, F.; et al. Taking account of context in population health intervention research: Guidance for producers, users and funders of research. Fac. Health Sci. Sport Res. Rep. 2018. [CrossRef] 